ESAIM: M2AN 46 (2012) 875-909

DOI: $10.1051 / \mathrm{m} 2 \mathrm{an} / 2011070$
ESAIM: Mathematical Modelling and Numerical Analysis

www.esaim-m2an.org

\title{
MATHEMATICAL AND NUMERICAL MODELLING OF PIEZOELECTRIC SENSORS
}

\author{
Sebastien Imperiale ${ }^{1,2}$ And PATRICK Joly ${ }^{2}$
}

\begin{abstract}
The present work aims at proposing a rigorous analysis of the mathematical and numerical modelling of ultrasonic piezoelectric sensors. This includes the well-posedness of the final model, the rigorous justification of the underlying approximation and the design and analysis of numerical methods. More precisely, we first justify mathematically the classical quasi-static approximation that reduces the electric unknowns to a scalar electric potential. We next justify the reduction of the computation of this electric potential to the piezoelectric domains only. Particular attention is devoted to the different boundary conditions used to model the emission and reception regimes of the sensor. Finally, an energy preserving finite element/finite difference numerical scheme is developed; its stability is analyzed and numerical results are presented.
\end{abstract}

Mathematics Subject Classification. 35L05, 35A35, 73R05, 35A40.

Received January 25, 2011. Revised July 8, 2011

Published online February 3, 2012.

\section{INTRODUCTION}

The present work has been achieved in the framework of a collaboration between the laboratory LIST of CEA Saclay and the Project Team POEMS which is common to INRIA, ENSTA and CNRS (UMR 7231), and motivated by an important application: non destructive testing by ultra-sound. The laboratory LIST is specialized in various aspects of non destructive testing, from both experimental and theoretical aspects, which includes numerical simulation which has now become a fundamental tool to understand and analyze the result of a non destructive testing experiment. The propagation of ultra-sonic elastic waves is often used to investigate the presence of defects in any manufactured item. In particular it is used for detecting defects (we mean a local heterogeneity, a geometrical imperfection, a crack, ...) inside metallic objects. As a fundamental and illustrative industrial application, we can cite the inspection of nuclear reactors.

Of course, to construct a reliable and efficient simulation tool in ultra-sonic non destructive testing, it is important to master the numerical simulation of elastic waves, for instance efficient discretization techniques for solving elastodynamic equations in a very general context: heterogeneous, anisotropic elastic (possibly viscoelastic) media. Nowadays, there are many satisfactory highly accurate numerical methods for the resolution of elastodynamic equations. In particular, at Project POEMS, we have intensively developed the technique of

Keywords and phrases. Piezoelectricity, quasi-static approximation, ultrasonic sensors.

1 CEA, List, Saclay, 91191 Gif-sur-Yvette, France. sebastien.imperiale@inria.fr

2 INRIA Rocquencourt, Domaine de Voluceau, Rocquencourt, 78153 Le Chesnay, France. patrick.joly@inria.fr 
spectral finite elements (with Gauss-Lobatto numerical integration) on general quadrilateral (in 2D) or hexahedral (in 3D) meshes (see $[4,6]$ ). These methods offer very good performance in terms of accuracy as well as computational efficiency and we have "naturally chosen" to use this method as the basic numerical tool for the "purely elastic part" of the modelling problem. It is important also to be able to deal with unbounded propagation media: of course, real objects are not unbounded but very often, one uses ultra-sound to investigate locally such a structure, namely in a domain which is small with respect to the size of the object which can then be seen as unbounded at this scale. Even though some important questions remain open from this point of view, in particular the treatment of some anisotropic media, the technique of perfectly matched layers offers a very satisfactory solution in most realistic applications.

A more challenging issue, which is more precisely the object of the present paper, is the mathematical and numerical modeling of the generation of ultra-sound, which is done by using special devices called piezoelectric transducers or sensors. These transducers are (partly) made of piezo-electric materials, namely elastic materials which have the astonishing property of "transforming" elastic waves (via the internal deformations they induce) into electromagnetic fields and related electric currents (the electric currents are the quantities that can be controlled and/or recorded), and reciprocally. As a consequence, such devices are used both for the generation of ultra-sound via an electric generator (this is the emission process) and for the recording of the electric currents associated to the "echos" i.e. the elastic waves reflected into the elastic medium, in particular by the possible defects. In more detail, piezoelectric sensors incorporate a periodic array of piezoelectric bars embedded in an elastic matrix and are in contact with the object under investigation. In the emission process, the piezoelectric bars can be energized (electrically) more or less independently from one another, which allows some flexibility in the nature of the wave that is sent in the elastic medium.

The numerical simulation of such devices is not a trivial issue. The first difficulty is the intrinsic complexity of the mathematical model that describes the behavior of piezoelectric models. The equations of piezoelectricity couple the equations of elastodynamics with Maxwell's equations. In the time dependent regime, an immediate and obvious difficulty is linked to the fact that the propagation velocity of elastic waves is by several orders of magnitude smaller than the speed of light which induces in the same model the co-existence of very different time and space scales that are almost impossible to tackle via a pure numerical approach. That is why a simplified (with respect to the numerical approximation) approximate model is required: this is the so-called quasi-static piezoelectric model, that is presented in many books of physics or publications (see $[5,8,12]$ ), very often with an argument which is difficult to understand for a mathematician. In this model, the electromagnetic unknowns (originally the electric and magnetic fields) are reduced to a scalar electric potential which is coupled to the displacement field in a mixed elliptic - hyperbolic system of equations in which the speed of light is considered as infinite.

Many engineers have proposed numerical approaches to the numerical modeling of piezoelectric sensors $([3,7,11,14]$ and more particularly $[1]$ which is more in the spirit of the present work). However, we did not find in the literature of applied mathematics any serious work (including the well-posedness of the final model, the rigorous justification of the underlying approximation and the design and analysis of numerical methods) in this direction for time dependent problem (see [13] for a mathematical analysis of general frequency dependent piezoelectric problems or [17] for the analysis of non-linear time dependent problems). The present work aims at proposing, at least partly, such an approach. To be more precise, the emphasis will be put on the following points which covers both mathematical modeling and numerical approximations issues:

- we justify rigorously the quasi-static approximation with error estimates. This is the topic of Section 3;

- in Section 4.1 we justify the fact that the effective computations of the electric potential, a priori defined in the whole space, can be reduced to the piezoelectric domain, by exploiting the strong contrast of dielectric permittivity between the piezoelectric domain and the elastic medium;

- in Section 4.2 we propose a modelling of the emission and reception processes via appropriate (non local) boundary conditions along the (very thin) electrodes attached to the piezoelectric sensors and establish the well-posedness of the fully coupled problem; 
- in Section 5 we present a space and time discretization procedure that is compatible with the use of spectral finite elements and whose stability is guaranteed theoretically;

- we present in Section 6 numerical computations corresponding to "realistic" situations.

\section{Preliminary notation}

First we recap the notation used in [9] Chapter 3, for writing the equations of elastodynamics and additional ones needed for the equations of piezoelectricity. The euclidean scalar product in $\mathbb{R}^{3}$ will be denoted

$$
u \cdot v=\sum_{i=1}^{3} u_{i} v_{i} \quad \forall(u, v) \in \mathbb{R}^{3} \times \mathbb{R}^{3} .
$$

A second order tensor is a linear mapping from $\mathbb{R}^{3}$ into itself, $\varepsilon=\left(\varepsilon_{i j}\right) \in \mathcal{L}\left(\mathbb{R}^{3}\right)$. In $\mathcal{L}\left(\mathbb{R}^{3}\right)$, we define the scalar product

$$
\sigma: \varepsilon=\sum_{i, j=1}^{3} \sigma_{i j} \varepsilon_{i j}, \quad \forall(\sigma, \varepsilon) \in \mathcal{L}\left(\mathbb{R}^{3}\right) \times \mathcal{L}\left(\mathbb{R}^{3}\right) .
$$

In the following, we shall use for simplicity the same notation $|\cdot|$ for denoting the norms associated to both inner products (2.1) and (2.2). In principle the context in which this notation will be used will eliminate any ambiguity.

The space of linear mappings from $\mathcal{L}\left(\mathbb{R}^{3}\right)$ into itself is denoted $\mathcal{L}^{2}\left(\mathbb{R}^{3}\right)$. Any element of $\mathcal{L}^{2}\left(\mathbb{R}^{3}\right)$ is associated to a fourth order tensor $\mathbf{C}=\left(\mathbf{C}_{i j k l}\right)$ such that

$$
(C \varepsilon)_{i j}=\sum_{k, l=1}^{3} \mathbf{C}_{i j k l} \varepsilon_{k l}
$$

To write the equations of piezoelectricity, we must introduce the space $\mathcal{L}\left(\mathbb{R}^{3}, \mathcal{L}\left(\mathbb{R}^{3}\right)\right)$ of linear mappings from $\mathbb{R}^{3}$ into $\mathcal{L}\left(\mathbb{R}^{3}\right)$ that transform vectors into second order tensors. They are associated with three indices tensors $\mathbf{d}=\left(\mathbf{d}_{k i j}\right)$ such that

$$
(\mathbf{d} u)_{i j}=\sum_{k=1}^{3} \mathbf{d}_{k i j} u_{k}
$$

The tranpose of $\mathbf{d}$, with respect to the inner products $(2.1)$ and $(2.2)$, namely $\mathbf{d}^{T}$, is the element of $\mathcal{L}\left(\mathcal{L}\left(\mathbb{R}^{3}\right), \mathbb{R}^{3}\right)$ (i.e. it transforms second order tensors into vectors) defined by

$$
\left(\mathbf{d}^{T} \varepsilon\right)_{k}=\sum_{i, j=1}^{3} \mathbf{d}_{k i j} \varepsilon_{i j} .
$$

Also we shall use the notation div $u$ for the scalar divergence of a vector field defined in $\mathbb{R}^{3}$ into $\mathbb{R}^{3}$ and the notation $\operatorname{div} \sigma$ for the vectorial divergence of a tensor field $\sigma: \mathbb{R}^{3} \rightarrow \mathcal{L}\left(\mathbb{R}^{3}\right)$. Let us recall that

$$
(\operatorname{div} \sigma)_{i}=\operatorname{div}\left(\sigma_{i}\right)
$$

if $\sigma_{i}$ denotes the $i$ th line vector of $\sigma$. 


\section{Piezoelectricity AND the quasi-Static Approximation}

\subsection{The equations of piezoelectricity}

\subsubsection{The full equations}

Even though this will not always be mentioned explicitly, all the unknowns in our model are functions of the position variable $x=\left(x_{1}, x_{2}, x_{3}\right) \in \mathbb{R}^{3}$ and the time $t>0$. In what follows, the piezoelectric material is supposed to occupy a bounded domain $\Omega_{S} \subset \mathbb{R}^{3}$ (S for solid). Therefore, all the "elastic unknowns" (displacements, deformations, stresses) will be defined for $x \in \Omega_{S}$ only while the "electric unknowns" (magnetic and electric fields) will be defined in the whole space.

As already mentioned, the equations of piezoelectricity result from a coupling with linear Maxwell's equations and linear elastodynamic equations. We start with the classical Maxwell linear equations

$$
\mu \frac{\partial}{\partial t} H+\nabla \times E=m, \quad \frac{\partial}{\partial t} D-\nabla \times H=\jmath, \quad x \in \mathbb{R}^{3}, \quad t>0 .
$$

Here the vector-valued unknowns are the magnetic field $H$, the electric displacement $D$ and the electric field $E$. The data $\mu=\mu(x) \in \mathcal{L}\left(\mathbb{R}^{3}\right)$ is the (tensor) magnetic permeability of the medium, which is a measurable function of $x$ that satisfies the usual symmetry, boundedness and uniform coercivity properties (with $0<\mu_{-} \leq \mu_{+}<$ $+\infty)$ :

$$
\text { a.e. } x \in \mathbb{R}^{3}, \quad \mu_{i j}(x)=\mu_{j i}(x), \quad \text { and } \quad \mu_{-}|u|^{2} \leq \mu(x) u \cdot u \leq \mu_{+}|u|^{2}, \quad \forall u \in \mathbb{R}^{3} \text {. }
$$

For the sake of generality, we have included volume sources, namely the vector fields $\jmath=\jmath(x, t)$ (an imposed electric current density) and $m=m(x, t)$ (an imposed magnetic current density).

The propagation of elastic waves obey the fundamental law of continuum mechanics, namely

$$
\rho \frac{\partial^{2}}{\partial t^{2}} u-\operatorname{div} \sigma=f, \quad x \in \mathbb{R}^{3}, \quad t>0,
$$

where the vector valued unknown $u$ represents the displacement of solid particles and the tensor valued unknown $\sigma$ represents the stress tensor at each point of the solid body. Let us recall that this tensor is symmetric

$$
\text { a.e. } x \in \mathbb{R}^{3}, \quad \sigma_{i j}(x, t)=\sigma_{i j}(x, t), \quad 1 \leq i, j \leq 3, \quad t>0 .
$$

The data $\rho=\rho(x)$ is the density of the material. It is a real valued measurable function satisfying:

$$
\text { a.e. } x \in \Omega_{S}, \quad 0<\rho_{-} \leq \rho(x) \leq \rho_{+}<+\infty \text {. }
$$

The vector field $f=f(x, t)$ is a (given) volumic density of sources.

To complete equations (3.1) and (3.3), we need to make precise the constitutive laws that govern the behavior of the material by expressing how the electric displacement $D$ and the stress tensor $\sigma$ are related to the displacement field $u$ and the electric field $E$. In the context of linear piezoelectricity, we have

$$
D=\epsilon E+\mathbf{d}^{T} e(u), \quad \sigma=\mathbf{C} e(u)-\mathbf{d} E, \quad t>0,
$$

where, under the usual assumption of small deformations, $e(u)=\left(e_{i j}(u)\right)$ is the linearized deformation tensor defined by

$$
e_{i j}(u)=\frac{1}{2}\left(\frac{\partial}{\partial x_{i}} u_{j}+\frac{\partial}{\partial x_{j}} u_{i}\right), \quad 1 \leq i, j \leq 3 .
$$

In (3.6), $\mathbf{C}=\mathbf{C}(x) \equiv\left(\mathbf{C}_{i j k l}(x)\right)$ (respectively $\left.\mathbf{d}=\mathbf{d}(x) \equiv\left(\mathbf{d}_{k i j}(x)\right)\right)$ is a function with values in $\mathcal{L}^{2}\left(\mathbb{R}^{3}\right)$ (respectively in $\mathcal{L}\left(\mathbb{R}^{3}, \mathcal{L}\left(\mathbb{R}^{3}\right)\right)$ ) called the elasticity tensor (respectively the piezoelectric tensor). By convention

$$
\mathbf{C}(x)=0 \quad \text { and } \quad \mathbf{d}(x)=0, \quad \text { if } x \notin \Omega_{S} .
$$


The elasticity tensor $\mathbf{C}=\mathbf{C}(x)$ satisfies the usual symmetry, boundedness and uniform coercivity properties (with $0<\mathbf{C}_{-} \leq \mathbf{C}_{+}<+\infty$ ):

$$
\text { a.e. } x \in \Omega_{S}, \quad \mathbf{C}_{i j k l}(x)=\mathbf{C}_{j i k l}(x)=\mathbf{C}_{k l i j}(x) \quad \text { and } \quad \mathbf{C}_{-}|\varepsilon|^{2} \leq \mathbf{C}(x) \varepsilon \cdot \varepsilon \leq \mathbf{C}_{+}|\varepsilon|^{2}, \quad \forall \varepsilon \in \mathcal{L}\left(\mathbb{R}^{3}\right) \text {. }
$$

The piezoelectric tensor $\mathbf{d}(x)$ satisfies the following symmetry properties (that ensure the symmetry of the stress tensor $\sigma)$ :

$$
\text { a.e. } x \in \Omega_{S}, \quad \mathbf{d}_{k i j}(x)=\mathbf{d}_{k j i}(x) \text {. }
$$

Finally $\epsilon=\epsilon(x) \in \mathcal{L}\left(\mathbb{R}^{3}\right)$ is the dielectric permittivity of the medium, which is a measurable function of $x$ that satisfies the usual symmetry, boundedness and uniform coercivity properties (with $0<\epsilon_{-} \leq \epsilon_{+}<+\infty$ ):

$$
\text { a.e. } x \in \mathbb{R}^{3}, \quad \epsilon_{i j}(x)=\epsilon_{j i}(x) \quad \text { and } \quad \epsilon_{-}|u|^{2} \leq \epsilon(x) u \cdot u \leq \epsilon_{+}|u|^{2}, \quad \forall u \in \mathbb{R}^{3} .
$$

Eliminating $D$ and $\sigma$ in function of $E$ and $u$ using (3.6), one finally derive the equations of piezoelectricity as a system of partial differential equations in $(E, H, u)$ :

$$
\begin{cases}\mu \frac{\partial}{\partial t} H+\nabla \times E=m, & x \in \mathbb{R}^{3}, \quad t>0 \\ \epsilon \frac{\partial}{\partial t} E-\nabla \times H+\frac{\partial}{\partial t} \mathbf{d}^{T} e(u)=\jmath, & x \in \mathbb{R}^{3}, \quad t>0 \\ \rho \frac{\partial^{2}}{\partial t^{2}} u-\operatorname{div} \mathbf{C} e(u)+\operatorname{div} \mathbf{d} E=f, & x \in \Omega_{S}, \quad t>0 .\end{cases}
$$

Of course, to get a well defined initial boundary value problem, (3.10) must be completed with initial conditions

$$
E(x, 0)=E^{0}(x), \quad H(x, 0)=H^{0}(x), \quad x \in \mathbb{R}^{3}, \quad u(x, 0)=u^{0}(x), \quad \frac{\partial}{\partial t} u(x, 0)=u^{1}(x), \quad x \in \Omega_{S},
$$

and by a boundary condition on $\partial \Omega_{S}$ relative to the displacement field $u$, for instance the free boundary condition (the most usual one)

$$
\sigma \mathbf{n}:=(\mathbf{C} e(u)-(\mathbf{d} E)) \mathbf{n}=0, \quad x \in \partial \Omega_{S},
$$

where $\mathbf{n}$ denotes the unit normal vector to $\partial \Omega_{S}$, outgoing with respect to $\Omega_{S}$.

Remark 3.1. For more generality, we could consider a non zero right hand side in (3.12), which would correspond to an imposed surfacic force density. One could also consider that one part of $\partial \Omega_{S}$ is clamped, that is to say that, along one part of $\partial \Omega_{S},(3.12)$ would be replaced by the Dirichlet condition $u=0$.

The mathematical theory of the initial boundary value problem (3.10)-(3.12) is rather straightforward. First, it is not difficult to check that, thanks to assumptions (3.2), (3.5), (3.7)-(3.9), this system is of hyperbolic nature. Next, one can easily derive a (formal) energy identity satisfied by any (smooth enough) solution of (3.10). Defining the total energy as the sum of the electromagnetic energy and the mechanical energy

$$
\begin{gathered}
\mathcal{E}(t)=\mathcal{E}_{e l}(t)+\mathcal{E}_{m e}(t) \\
\mathcal{E}_{e l}(t)=\frac{1}{2} \int_{\mathbb{R}^{3}}\left(\epsilon|E|^{2}+\mu|H|^{2}\right) \mathrm{d} x, \quad \mathcal{E}_{m e}(t)=\frac{1}{2} \int_{\Omega_{S}}\left(\rho\left|\frac{\partial}{\partial t} u\right|^{2}+\mathbf{C} e(u): e(u)\right) \mathrm{d} x,
\end{gathered}
$$

one easily derive by the usual technique (the details are left to the reader) the energy identity

$$
\frac{\mathrm{d}}{\mathrm{d} t} \mathcal{E}(t)=\int_{\mathbb{R}^{3}}(\jmath \cdot E+m \cdot H) \mathrm{d} x+\int_{\Omega_{S}} f \cdot \frac{\partial}{\partial t} u \mathrm{~d} x,
$$


which leads, via Gronwall's lemma, to a priori estimates that constitute the basis of the existence and uniqueness theory, namely

$$
\mathcal{E}(t)^{\frac{1}{2}} \leq \mathcal{E}(0)^{\frac{1}{2}}+\int_{0}^{t}\left(\int_{\mathbb{R}^{3}}\left|\epsilon^{-\frac{1}{2}} \jmath(\cdot, s)\right|^{2}+\left|\mu^{-\frac{1}{2}} m(\cdot, s)\right|^{2} \mathrm{~d} x+\int_{\Omega_{S}} \rho^{-1}|f(\cdot, s)|^{2} \mathrm{~d} x\right)^{\frac{1}{2}} \mathrm{~d} s .
$$

More precisely, the problem (3.10)-(3.12) can easily be put in the framework of Hille-Yosida's theory and this is a simple exercise on the use of this theory to obtain the following theorem:

Theorem 3.1. Assume that the initial data have the following regularity

$$
\left(E_{0}, H_{0}\right) \in H\left(\operatorname{rot}, \mathbb{R}^{3}\right) \times H\left(\operatorname{rot}, \mathbb{R}^{3}\right) \quad\left(u_{0}, u_{1}\right) \in H^{1}\left(\Omega_{S}\right)^{3} \times H^{1}\left(\Omega_{S}\right)^{3},
$$

$$
\operatorname{div} \mathbf{C} e\left(u_{0}\right)-\operatorname{div} \mathbf{d} E_{0} \in L^{2}\left(\Omega_{S}\right)^{3},
$$

and that the source terms satisfy

$$
m \in C^{0}\left(\mathbb{R}^{+} ; L^{2}\left(\mathbb{R}^{3}\right)^{3}\right), \quad \jmath \in C^{0}\left(\mathbb{R}^{+} ; L^{2}\left(\mathbb{R}^{3}\right)^{3}\right), \quad f \in C^{1}\left(\mathbb{R}^{+} ; L^{2}\left(\Omega_{S}\right)^{3}\right) .
$$

Then the problem (3.10)-(3.12) admits a unique strong solution $(E, H, u)$ satisfying

$$
\left\{\begin{array}{l}
E \in C^{1}\left(\mathbb{R}^{+} ; L^{2}\left(\mathbb{R}^{3}\right)^{3}\right) \cap C^{0}\left(\mathbb{R}^{+} ; H\left(r o t, \mathbb{R}^{3}\right)\right), \\
H \in C^{1}\left(\mathbb{R}^{+} ; L^{2}\left(\mathbb{R}^{3}\right)^{3}\right) \cap C^{0}\left(\mathbb{R}^{+} ; H\left(\operatorname{rot}, \mathbb{R}^{3}\right)\right), \\
u \in C^{2}\left(\mathbb{R}^{+} ; L^{2}\left(\Omega_{S}\right)^{3}\right) \cap C^{1}\left(\mathbb{R}^{+} ; H^{1}\left(\Omega_{S}\right)^{3}\right) .
\end{array}\right.
$$

\subsection{The quasi-static piezoelectric equations and their mathematical justification}

\subsubsection{Quasi-static piezoelectric equations}

If one tries to solve numerically the problem (3.10), one has to deal with two wave propagation phenomena with completely different velocities. Indeed the full piezoelectric equations couple an electromagnetic propagation $\left(>10^{8} \mathrm{~m} / \mathrm{s}\right)$ with an elastodynamic propagation $\left(<10^{4} \mathrm{~m} / \mathrm{s}\right)$. To handle this problem physicists have introduced the quasi static approximation $[5,8]$. It consists to consider that the electric field derives from a scalar electric potential $\varphi$, namely that

$$
E=-\nabla \varphi
$$

Substituting such an equality in the last two equations of (3.10) gives

$$
\begin{cases}\epsilon \frac{\partial}{\partial t} \nabla \varphi+\nabla \times H-\frac{\partial}{\partial t} \mathbf{d}^{T} e(u)=-\jmath, & x \in \mathbb{R}^{3}, \quad t>0 \\ \rho \frac{\partial^{2}}{\partial t^{2}} u-\operatorname{div} \mathbf{C} e(u)-\operatorname{div} \mathbf{d} \nabla \varphi=f, & x \in \Omega_{S}, \quad t>0 .\end{cases}
$$

We can eliminate the magnetic field (which does not appear in the second equation) by taking the divergence of the first equation. After integration in time, setting

$$
J(x, t)=-\int_{0}^{t} \jmath(x, s) \mathrm{d} s
$$

we obtain the quasi-static piezoelectric model, as it can be found in many book of physics,

$$
\begin{cases}\operatorname{div}(\epsilon \nabla \varphi)-\operatorname{div}\left(\mathbf{d}^{T} e(u)\right)=\operatorname{div}\left(J+\epsilon E_{0}-\mathbf{d}^{T} e\left(u_{0}\right)\right), & x \in \mathbb{R}^{3}, \quad t>0, \\ \rho \frac{\partial^{2}}{\partial t^{2}} u-\operatorname{div} \mathbf{C} e(u)-\operatorname{div} \mathbf{d} \nabla \varphi=f, & x \in \Omega_{S}, \quad t>0,\end{cases}
$$


which can be seen as the coupling between an elliptic equation for the electric potential (the first equation in (3.22), $u$ contributing to the source term) with an hyperbolic system for the displacement field $u$ (the second equation in (3.22), $\varphi$ contributing to the source term).

To complete (3.22), we only need initial conditions for the displacement field:

$$
u(x, 0)=u_{0}(x), \quad \frac{\partial}{\partial t} u(x, 0)=u_{1}(x)
$$

and the free boundary condition which becomes ( $c f .(3.12)$ and (3.21))

$$
(\mathbf{C} e(u)-(\mathbf{d} \nabla \varphi)) \mathbf{n}=0 \quad x \in \partial \Omega_{S} .
$$

The existence and uniqueness theory of (3.22)-(3.24) is again rather straightforward. We simply need to work in the functional framework adapted to the electrostatic part of the problem, i.e. to the resolution of the (generalized) Laplace equation in $\mathbb{R}^{3}$.

That is why we introduce the Beppo-Levi space

$$
W^{1}\left(\mathbb{R}^{3}\right)=\left\{\psi \in H_{l o c}^{1}\left(\mathbb{R}^{3}\right) / \frac{\psi}{\left(1+|x|^{2}\right)^{\frac{1}{2}}} \in L^{2}\left(\mathbb{R}^{3}\right), \nabla \psi \in L^{2}\left(\mathbb{R}^{3}\right)^{3}\right\},
$$

equipped with its natural Hilbert space norm,

$$
\|\psi\|_{W^{1}\left(\mathbb{R}^{3}\right)}^{2}=\int_{\mathbb{R}^{3}} \frac{|\psi|^{2}}{1+|x|^{2}} \mathrm{~d} x+\int_{\mathbb{R}^{3}}|\nabla \psi|^{2} \mathrm{~d} x
$$

and the associated quotient space (introduced to take into account that the electric potential is only defined up to an additive constant)

$$
\mathbf{W}=W^{1}\left(\mathbb{R}^{3}\right) / \mathbb{R} .
$$

Thanks to Hardy's inequality, we can equip W with the following (adapted) Hilbert space norm

$$
\|\psi\|_{\mathbf{W}}^{2}:=\int_{\mathbb{R}^{3}}(\epsilon \nabla \psi, \nabla \psi) \mathrm{d} x .
$$

Theorem 3.2. Assuming that

$$
\left(u_{0}, u_{1}\right) \in H^{1}\left(\Omega_{S}\right)^{3} \times L^{2}\left(\Omega_{S}\right)^{3}, \quad E_{0} \in L^{2}\left(\mathbb{R}^{3}\right)^{3}
$$

and

$$
f \in C^{1}\left(\mathbb{R}^{+} ; L^{2}\left(\Omega_{S}\right)^{3}\right), \quad \jmath \in C^{0}\left(\mathbb{R}^{+} ; L^{2}\left(\mathbb{R}^{3}\right)^{3}\right)
$$

the problem (3.22)-(3.24) admits a unique strong solution $(\varphi, u)$ such that

$$
\varphi \in C^{1}\left(\mathbb{R}^{+} ; W\left(\mathbb{R}^{3}\right) / \mathbb{R}\right) \quad u \in C^{2}\left(\mathbb{R}^{+} ; L^{2}\left(\Omega_{S}\right)^{3}\right) \cap C^{1}\left(\mathbb{R}^{+} ; H^{1}\left(\Omega_{S}\right)^{3}\right) .
$$

Proof. We give the main steps of the proof (the details will be left to the reader) which we find instructive to analyze qualitatively the piezoelectric effects (see the paragraph which follows this proof) and motivates the numerical method that we shall propose later.

We first give a weak form (or variational formulation) of (3.22)-(3.24). Let us set $\mathbf{V}=H^{1}\left(\Omega_{S}\right)^{3}$ and introduce the continuous bilinear form defined on $\mathbf{V} \times \mathbf{W}$ by

$$
b(v, \psi):=\int_{\Omega_{S}} e(u): \mathbf{d} \nabla \psi \mathrm{d} x \equiv \int_{\Omega_{S}} \mathbf{d}^{T} e(u) \cdot \nabla \psi \mathrm{d} x, \quad \forall(v, \psi) \in \mathbf{V} \times \mathbf{W} .
$$


We also define the positive symmetric and continuous bilinear form in $\mathbf{V}$

$$
a(u, v)=\int_{\mathbb{R}^{3}} \mathbf{C} e(u): e(v) \mathrm{d} x, \quad \forall(u, v) \in \mathbf{V} \times \mathbf{V} .
$$

Finally, we consider the (weighted) $L^{2}$ inner product in $\mathbf{H}:=L^{2}\left(\Omega_{S}\right)^{3} \subset \mathbf{V}$ (with $\mathbf{H}$ dense in $\mathbf{V}$ )

$$
(u, v)_{\mathbf{H}}=\int_{\mathbb{R}^{3}} \rho u \cdot v \mathrm{~d} x, \quad \forall(u, v) \in \mathbf{H} \times \mathbf{H} .
$$

Solving amounts to find $u: \mathbb{R}^{+} \longrightarrow \mathbf{V}$ and $\varphi: \mathbb{R}^{+} \longrightarrow \mathbf{W}$ such that

$$
\begin{cases}(\varphi(t), \psi)_{\mathbf{W}}-b(u(t), \psi)=\langle L(t), \psi\rangle, & \forall \psi \in \mathbf{W}, \\ \frac{\mathrm{d}^{2}}{\mathrm{~d} t^{2}}(u(t), v)_{\mathbf{H}}+a(u(t), v)+b(v, \varphi(t))=\langle F(t), v\rangle, & \forall v \in \mathbf{V}, \\ u(0)=u_{0}, \quad \frac{\mathrm{d} u}{\mathrm{~d} t}(0)=u_{1}, & \end{cases}
$$

where $L(t)$ and $F(t)$ are linear forms on $\mathbf{W}$ and $\mathbf{V}$ respectively, defined by

$$
\langle L(t), \psi\rangle=\int_{\mathbb{R}^{3}}\left(J(t)+\epsilon E_{0}-\mathbf{d}^{T} e\left(u_{0}\right)\right) \cdot \nabla \psi \mathrm{d} x, \quad \forall \psi \in \mathbf{W}, \quad\langle F(t), \psi\rangle=\int_{\Omega_{S}} f(t) \cdot v \mathrm{~d} x, \quad \forall v \in \mathbf{V} .
$$

Next, we eliminate $\varphi$ to get an evolution equation for $u$ only. To do so, we use Riesz' theorem to introduce the operator $\mathbf{B} \in \mathcal{L}(\mathbf{W}, \mathbf{V})$ and $\mathbf{L}(\mathbf{t}) \in \mathbf{V}$ such that

$$
b(v, \psi):=(\mathbf{B} v, \psi)_{\mathbf{W}} \quad \forall(v, \psi) \in \mathbf{V} \times \mathbf{W}, \quad\langle L(t), \psi\rangle=(\mathbf{L}(t), \psi), \quad \forall \psi \in \mathbf{W} .
$$

The first equation of (3.32) gives simply

$$
\varphi(t)=\mathbf{B} u(t)+\mathbf{L}(t),
$$

that we can substitute into the second equation of (3.32) to see that $u$ is solution of the evolution problem

$$
\left\{\begin{array}{l}
\frac{\mathrm{d}^{2}}{\mathrm{~d} t^{2}}(u(t), v)_{\mathbf{H}}+a^{*}(u(t), v)=\langle F(t), v\rangle-b(v, \mathbf{L}(t)), \quad \forall \psi \in \mathbf{W}, \\
u(0)=u_{0}, \quad \frac{\mathrm{d} u}{\mathrm{~d} t}(0)=u_{1},
\end{array}\right.
$$

where $a^{*}(\cdot, \cdot)$ is the symmetric positive (even coercive thanks to Korn's inequality) and continuous bilinear form defined on $\mathbf{V}$ by

$$
a^{*}(u, v)=a(u, v)+(\mathbf{B} u, \mathbf{B} v)_{\mathbf{V}}, \quad \forall(u, v) \in \mathbf{V} \times \mathbf{V} .
$$

We have now put our problem in a classical abstract framework. we can for instance apply Hille-Yosida's theory by introducing the positive unbounded selfadjoint operator $\mathbf{A}^{*}$ in $\mathbf{H}$ defined by

$$
\begin{gathered}
D\left(\mathbf{A}^{*}\right)=\left\{u \in \mathbf{V} / \exists C(u)>0 \text { such that }\left|a^{*}(u, v)\right| \leq C(u)\|v\|_{\mathbf{H}}, \quad \forall v \in \mathbf{V}\right\}, \\
\forall u \in D\left(\mathbf{A}^{*}\right), \quad \mathbf{A}^{*} u \in \mathbf{H} \text { is defined by }\left(\mathbf{A}^{*} u, v\right)_{\mathbf{H}}=a^{*}(u, v), \quad \forall v \in \mathbf{V},
\end{gathered}
$$

then (3.32) is equivalent to the abstract evolution equation in $\mathbf{H}$ :

$$
\frac{\mathrm{d}^{2} u}{\mathrm{~d} t^{2}}+\mathbf{A}^{*} u=g(t), \quad u(0)=u_{0}, \quad \frac{\mathrm{d} u}{\mathrm{~d} t}(0)=u_{1},
$$


where $g(t) \in C^{1}\left(\mathbb{R}^{+} ; \mathbf{H}\right)$ (we omit the details). Then, the existence and uniqueness result is a direct consequence of Hille-Yosida's theorem, after checking that

$$
D\left(\mathbf{A}^{*}\right)=\left\{u \in H^{1}\left(\Omega_{S}\right)^{3} / \operatorname{div}(\mathbf{C} e(u)+\mathbf{d} \nabla \varphi(u)) \in L^{2}\left(\Omega_{S}\right)^{3},(\mathbf{C} e(u)-(\mathbf{d} \nabla \varphi(u))) \mathbf{n}=0, \quad x \in \partial \Omega_{S}\right\},
$$

where $\varphi(u) \in W^{1}\left(\mathbb{R}^{3}\right) / \mathbb{R}$ is defined as the unique solution of the generalized Laplace problem

$$
\operatorname{div}(\epsilon \nabla \varphi(u))=\operatorname{div}\left(\mathbf{d}^{T} e(u)\right)+\operatorname{div}\left(J+\epsilon E_{0}-\mathbf{d}^{T} e\left(u_{0}\right)\right) \quad x \in \mathbb{R}^{3} .
$$

Looking at (3.33) and (3.34), we see that, if one reduces the problem to the displacement field $u$, passing from the pure elastic case to the piezoelectric amounts essentially to replace the classical elastic stiffness bilinear form $a(u, v)$ by the augmented stiffness bilinear form $a^{*}(u, v)$, the electric effects being represented by the additional term $(\mathbf{B} u, \mathbf{B} v)_{\mathbf{V}}$. Note however that this term is a non local one since the operator $\mathbf{B}$ can be reinterpreted as

$$
\mathbf{B} u=\varphi(u) \quad \text { where } \varphi(u) \in W^{1}\left(\mathbb{R}^{3}\right) / \mathbb{R} \quad \text { is the unique solution of (3.35). }
$$

As a consequence, the solutions of (3.22) propagate with infinite propagation velocity, which reflects the fact that the speed of light has been considered as infinite in Maxwell's equations.

As for the full model, there is a natural energy associated to (3.22)

$$
\mathcal{E}_{q s}(t)=\frac{1}{2} \int_{\mathbb{R}^{3}}\left|\epsilon^{\frac{1}{2}} \nabla \varphi\right|^{2} \mathrm{~d} x+\frac{1}{2} \int_{\Omega_{S}}\left(\rho\left|\frac{\partial}{\partial t} u\right|^{2}+\mathbf{C} e(u): e(u)\right) \mathrm{d} x,
$$

that satisfies the identity

$$
\frac{\mathrm{d}}{\mathrm{d} t} \mathcal{E}_{q s}(t)=-\int_{\mathbb{R}^{3}} \jmath \cdot \nabla \varphi \mathrm{d} x+\int_{\Omega_{S}} f \cdot \frac{\partial}{\partial t} u \mathrm{~d} x,
$$

which leads to the a priori estimate

$$
\mathcal{E}_{q s}(t)^{\frac{1}{2}} \leq \mathcal{E}_{q s}(0)^{\frac{1}{2}}+\int_{0}^{T}\left(\int_{\mathbb{R}^{3}}\left|\epsilon^{-\frac{1}{2}} \jmath(\cdot, s)\right|^{2} \mathrm{~d} x+\int_{\Omega_{S}} \rho^{-1}|f(\cdot, s)|^{2} \mathrm{~d} x\right)^{\frac{1}{2}} \mathrm{~d} s .
$$

\subsubsection{Non-dimensionalization of the equations and formal derivation of the quasi-static model}

In this section we are going to show how one can formally derive (3.32) from (3.10) without invoking physical intuition. For this, it is useful to work with dimensionless unknowns, dimensionless variables and dimensionless coefficients. That is why we introduce reference time and length scales $L$ and $T$, to be fixed later, and rewrite the unknowns $(E, H, u)$ of $(3.10)$ as

$$
E(x, t)=E^{*} E_{r}\left(\frac{x}{L}, \frac{t}{T}\right), \quad H(x, t)=H^{*} H_{r}\left(\frac{x}{L}, \frac{t}{T}\right), \quad u(x, t)=L u_{r}\left(\frac{x}{L}, \frac{t}{T}\right),
$$

where the subscript $r$ refers to non-dimensionalized (or relative) quantities. We have used the length $L$ to nondimensionalize the displacement field $u$. The scalar quantities $E^{*}>0$ and $H^{*}>0$, used to scale $E$ and $H$, are respectively homogeneous to an electric and magnetic field: they will be fixed later.

We shall also work with relative coefficients. For the electric unknowns we shall write, as usual,

$$
\epsilon(x)=\epsilon_{0} \epsilon_{r}\left(\frac{x}{L}\right), \quad \mu(x)=\mu_{0} \mu_{r}\left(\frac{x}{L}\right),
$$

where $\epsilon_{0}$ and $\mu_{0}$ are respectively the electric permittivity and the magnetic permeability in the vacuum and $\epsilon_{r}$ and $\mu_{r}$ are the (dimensionless) relative electric permittivity and the magnetic permeability. Let us recall that the speed of light is defined by

$$
c_{0}=\left(\epsilon_{0} \mu_{0}\right)^{-\frac{1}{2}} .
$$


For the elastic unknowns, it is useful to introduce, for each $x \in \Omega_{S}$ and unit vector $\nu(|\nu|=1)$ the (strictly positive) eigenvalues $\left\{\lambda_{j}(x, \nu), j=1,2,3\right\}$ of the tensor $\mathbf{C}(x) \nu$ so that the quantities

$$
V_{j}(x, \nu):=\left(\frac{\lambda_{j}(x, \nu)}{\rho(x)}\right)^{\frac{1}{2}}, \quad 1 \leq j \leq 3,
$$

represent (locally) the velocities of elastic plane waves, more precisely, these are the phase velocities of the plane waves that would propagate in the direction $\nu$ in a homogeneous medium whose density and elasticity tensor would be equal to $\rho(x)$ and $\mathbf{C}(x)$. We then set

$$
\rho_{+}=\sup _{x \in \Omega_{S}} \rho(x), \quad V_{+}=\sup _{x \in \Omega_{S}} \sup _{|\nu|=1} \max _{1 \leq j \leq 3} V_{j}(x, \nu)
$$

and we scale $\rho$ and $\mathbf{C}$ as follows:

$$
\rho(x)=\rho_{+} \rho_{r}\left(\frac{x}{L}\right), \quad \mathbf{C}(x)=\rho_{+} V_{+}^{2} \mathbf{C}_{r}\left(\frac{x}{L}\right)
$$

so that the relative density $\rho_{r}$ and elasticity tensor $\mathbf{C}_{r}$ are dimensionless quantities.

It remains to non-dimensionalize the piezoelectric tensor $\mathbf{d}(x)$, which can be done as follows:

$$
\mathbf{d}(x)=d_{+} \mathbf{d}_{r}\left(\frac{x}{L}\right), \quad \text { where } d_{+}=\sup _{x \in \Omega_{S}}\left(\left|\mathbf{d}(x)^{T} \mathbf{d}(x)\right|_{2}\right)^{\frac{1}{2}} .
$$

Next, we deduce from (3.10) the equations satisfied by the dimensionless fields $\left(E_{r}, H_{r}, u_{r}\right)$ (for simplicity, we still denote $x$ and $t$ the scaled space and time variables and $\left.\widehat{\Omega}_{S}=\left\{x / L, x \in \Omega_{S}\right\}\right)$

$$
\begin{cases}\mu_{r} \frac{\partial}{\partial t} H_{r}+\left[\frac{V_{+} T}{L}\right]\left[\frac{\sqrt{\epsilon_{0}} E^{*}}{\sqrt{\mu_{0}} H^{*}}\right]\left[\frac{c_{0}}{V_{+}}\right] \nabla \times E_{r}=m_{r}, & x \in \mathbb{R}^{3}, \quad t>0, \\ \epsilon_{r} \frac{\partial}{\partial t} E_{r}-\left[\frac{V_{+} T}{L}\right]\left[\frac{\sqrt{\mu_{0}} H^{*}}{\sqrt{\epsilon_{0}} E^{*}}\right]\left[\frac{c_{0}}{V_{+}}\right] \nabla \times H_{r}+\left[\frac{d_{+}}{\epsilon_{0} E^{*}}\right] \frac{\partial}{\partial t} \mathbf{d}_{r}^{T} e\left(u_{r}\right)=\jmath_{r}, & x \in \mathbb{R}^{3}, \quad t>0, \\ \rho_{r} \frac{\partial^{2}}{\partial t^{2}} u_{r}-\left[\frac{V_{+}^{2} T^{2}}{L^{2}}\right] \operatorname{div} \mathbf{C}_{r} e\left(u_{r}\right)+\left[\frac{T^{2} d_{+} E^{*}}{L^{2} \rho_{+}}\right] \operatorname{div} \mathbf{d}_{r} E_{r}=f_{r}, & x \in \widehat{\Omega}_{S}, \quad t>0,\end{cases}
$$

where $m_{r}, \jmath_{r}$ and $f_{r}$ are scaled dimensionless source terms whose explicit expression is not needed for our purpose.

In (3.46), the reader will check easily that each coefficient between brackets is dimensionless. Let us now make the important choice of the length and time scales $L$ and $T$. As it is natural, we choose $L$ as the characteristic size of the bounded domain $\Omega_{S}$ and $T$ as the typical time for an elastic wave to travel along the distance $L$, which corresponds to

$$
L=V_{+} T \quad\left(\Longrightarrow \frac{V_{+} T}{L}=1\right) .
$$

This is where we express that we want to observe the physical phenomenon at a time scale that correspond to the propagation of elastic waves.

Less important is the choice of $E^{*}$ and $H^{*}$ (which simply correspond to a choice of unit to compute the electric field and magnetic field) which we adopt for simplifying as far as possible equations (3.46). More precisely, we choose $E^{*}$ and $H^{*}$ in such a way that (we symmetrize in particular the coupling terms)

$$
\sqrt{\epsilon_{0}} E^{*}=\sqrt{\mu_{0}} H^{*} \text { and } \quad \frac{T^{2} d_{+} E^{*}}{L^{2} \rho_{+}}=\frac{d_{+}}{\epsilon_{0} E^{*}} .
$$


We can then rewrite (3.46) with two additional dimensionless coefficients

$$
\gamma=\frac{d_{+}}{V_{+} \sqrt{\epsilon_{0} \rho_{+}}} \quad \text { and } \quad \delta=\frac{V_{+}}{c_{0}} \ll 1 .
$$

as follows

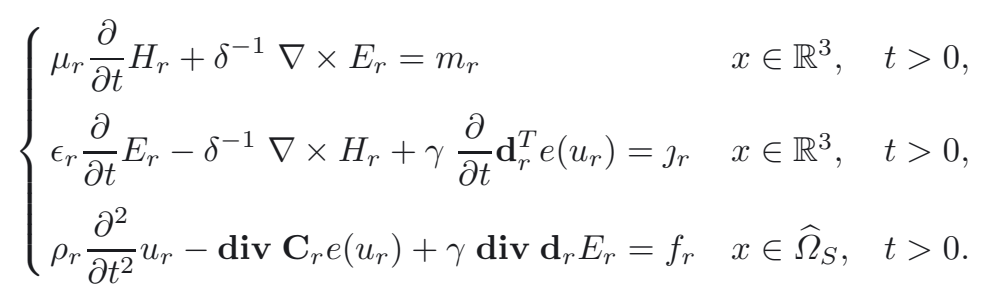

Remark 3.2. In contrast to $\delta$, which is less than $10^{-4}$, one can check that $\gamma$ is of the order of unity. Indeed, typical realistic values for the parameters $d_{+}, V_{+}$and $\rho_{+}$are $($see $[5,8])$ :

$$
d_{+} \simeq 10 \mathrm{C} / \mathrm{m}^{2} . \quad \rho_{+} \simeq 7500 \mathrm{~kg} / \mathrm{m}^{3}, \quad V_{+} \simeq 4500 \mathrm{~m} / \mathrm{s}
$$

which gives $\gamma \simeq 8$. since $\epsilon_{0}=1 /\left(36 \pi 10^{9}\right)$.

It is then easy to see how to obtain (3.22) formally. Rewriting the first equation of (3.10) as

$$
\nabla \times E_{r}=\delta\left(m_{r}-\mu_{r} \frac{\partial}{\partial t} H_{r}\right),
$$

then, assuming that $\frac{\partial}{\partial t} H_{r}$ remains bounded (see Rem. 3.3), then in the limit $\delta \rightarrow 0$

$$
\nabla \times E_{r}=0
$$

i.e. there exists a scalar potential $\varphi_{r}$ such that

$$
E_{r}=-\nabla \varphi_{r}
$$

Then, we can proceed as at the beginning of this section to derive the quasistatic equations (3.22).

Remark 3.3. We shall see in the next section that obtaining such a bound will be subject to appropriate assumptions on the data of the problem, in particular the initial conditions.

\subsection{Rigorous justification of the quasistatic model}

In this section, we consider $\delta$ as a varying parameter tending to 0 and wish to study the behavior of the solution $\left(E_{r}^{\delta}, H_{r}^{\delta}, u_{r}^{\delta}\right)$ of

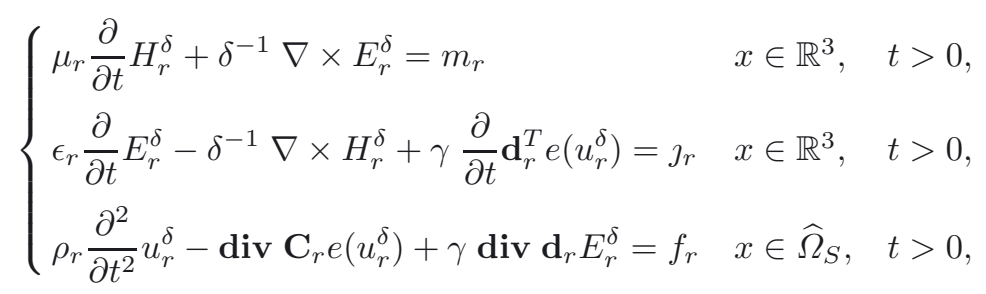

with initial conditions

$$
E_{r}^{\delta}(x, 0)=E_{r}^{0}(x), \quad H_{r}^{\delta}(x, 0)=H_{r}^{0}(x), \quad x \in \mathbb{R}^{3}, \quad u_{r}^{\delta}(x, 0)=u_{r}^{0}(x), \quad \frac{\partial}{\partial t} u_{r}^{\delta}(x, 0)=u_{r}^{1}(x), \quad x \in \widehat{\Omega}_{S}
$$


and the free boundary condition

$$
\left(\mathbf{C}_{r} e\left(u_{r}^{\delta}\right)-\left(\mathbf{d}_{r} E_{r}^{\delta}\right)\right) \mathbf{n}=0 \quad \text { on } \partial \widehat{\Omega}_{S},
$$

that we want to compare to the solution $\left(\varphi_{r}, u_{r}\right)$ of the corresponding limit problem

$$
\begin{cases}\operatorname{div}\left(\epsilon_{r} \nabla \varphi_{r}\right)-\gamma \operatorname{div}\left(\mathbf{d}_{r}^{T} e\left(u_{r}\right)\right)=\operatorname{div}\left(J_{r}+\epsilon E_{r}^{0}-\gamma \mathbf{d}_{r}^{T} e\left(u_{r}^{0}\right)\right), & x \in \mathbb{R}^{3}, \quad t>0, \\ \rho_{r} \frac{\partial^{2}}{\partial t^{2}} u_{r}-\operatorname{div} \mathbf{C}_{r} e\left(u_{r}\right)-\gamma \operatorname{div} \mathbf{d}_{r} \nabla \varphi_{r}=f_{r}, & x \in \widehat{\Omega}_{S}, \quad t>0,\end{cases}
$$

with the initial conditions

$$
u_{r}(x, 0)=u_{r}^{0}(x), \quad \frac{\partial}{\partial t} u_{r}(x, 0)=u_{r}^{1}(x), \quad x \in \widehat{\Omega}_{S},
$$

and the free boundary condition

$$
\left(\mathbf{C}_{r} e\left(u_{r}\right)-\left(\mathbf{d}_{r} \nabla \varphi_{r}\right)\right) \mathbf{n}=0 \quad x \in \partial \widehat{\Omega}_{S}
$$

In (3.55), we have set

$$
J_{r}(x, t)=-\int_{0}^{t} J_{r}(x, s) \mathrm{d} s
$$

At first glance, one would expect that

$$
\left(E_{r}^{\delta}, u_{r}^{\delta}\right) \quad \longrightarrow \quad\left(-\nabla \varphi_{r}, u_{r}\right) \quad \text { when } \delta \longrightarrow 0 .
$$

This is what happens under certain conditions that correspond to well prepared data.

Well prepared data. In this paragraph, we explain the conditions on the data of the problem that we shall assume in order to justify rigorously the quasistatic-model. Some of these assumptions are of pure technical nature (in particular those that refer to the smoothness of the initial data and source terms). Other assumptions are more fundamental. In particular, to obtain (3.58), we need to start, for the electromagnetic unknowns, from an electrostatic state, which corresponds to

$$
E_{r}^{0}=-\nabla \varphi_{r}^{0}, \quad \varphi_{r}^{0} \in W^{1}\left(\mathbb{R}^{3}\right) / \mathbb{R},
$$

which ensures that, at time $t=0, \nabla \times E_{r}^{\delta}(\cdot, 0)=0$. Moreover, for reasons that will clearly appear in the convergence proof (see Rem. 3.4), we shall also need that the initial magnetic field satisfies the magnetostatic condition:

$$
\nabla \times H_{r}^{0}=0 .
$$

To be in the framework of the existence result for strong solutions, we simply need that:

$$
\left(u_{r}^{0}, u_{r}^{1}\right) \in H^{1}\left(\widehat{\Omega}_{S}\right)^{3} \times H^{1}\left(\widehat{\Omega}_{S}\right)^{3}, \quad\left(\jmath_{r}, m_{r}, f_{r}\right) \in C_{l o c}^{0}\left(\mathbb{R}^{+} ; L^{2}\left(\mathbb{R}^{3}\right)^{3}\right) .
$$

In fact, we shall need also that $\frac{\partial}{\partial t} \nabla \times E_{r}^{\delta}(\cdot, 0)=0$ which imposes that

$$
\nabla \times m_{r}(\cdot, 0)=0, \quad \nabla \times\left[\epsilon_{r}^{-1}\left(J_{r}(\cdot, 0)+\gamma \mathbf{d}_{r} e\left(u_{r}^{1}\right)\right)\right]=0 .
$$

Finally, the other assumptions are made in order to ensure a sufficient regularity of the solution of (3.52). More precisely, we shall assume that

$$
\begin{aligned}
& \left(\jmath_{r}, m_{r}, f_{r}\right) \in W_{l o c}^{2,1}\left(\mathbb{R}^{+} ; L^{2}\left(\mathbb{R}^{3}\right)^{3}\right) \quad\left(\subset C^{1}\left(\mathbb{R}^{+} ; L^{2}\left(\mathbb{R}^{3}\right)^{3}\right)\right), \\
& \rho_{r}^{-1}\left[\operatorname{div}\left(\mathbf{C}_{r} e\left(u_{r}^{0}\right)-\gamma \mathbf{d}_{r} \nabla \varphi_{r}^{0}\right)+f_{r}(\cdot, 0)\right] \in H^{1}\left(\widehat{\Omega}_{S}\right)^{3}, \\
& \operatorname{div}\left[\mathbf{C}_{r} e\left(u_{r}^{1}\right)+\gamma^{2} \mathbf{d}_{r}^{T} \epsilon_{r}^{-1} \mathbf{d}_{r} e\left(u_{r}^{1}\right)-\gamma \mathbf{d}_{r}^{T} \epsilon_{r}^{-1} \jmath_{r}(\cdot, 0)\right] \in L^{2}\left(\widehat{\Omega}_{S}\right)^{3} .
\end{aligned}
$$


These additional assumptions ensure (this is a classical result that we shall admit here) that the solution has the additional regularity

$$
u_{r}^{\delta} \in C^{3}\left(\mathbb{R}^{+} ; L^{2}\left(\widehat{\Omega}_{S}\right)^{3}\right) \cap C^{2}\left(\mathbb{R}^{+} ; H^{1}\left(\widehat{\Omega}_{S}\right)^{3}\right), \quad\left(E_{r}^{\delta}, H_{r}^{\delta}\right) \in C^{2}\left(\mathbb{R}^{+} ; L^{2}\left(\mathbb{R}^{3}\right)^{3}\right) \cap C^{1}\left(\mathbb{R}^{+} ; H\left(\operatorname{rot} ; \mathbb{R}^{3}\right)\right) .
$$

In fact the key tool in the analysis is an appropriate Helmholtz decomposition of vector fields in $\mathbb{R}^{3}$. This is a trivial adaptation of classical results (see [15]):

Proposition 3.1. Any vector field $F \in L^{2}\left(\mathbb{R}^{3}\right)^{3}$ admits a unique decomposition as

$$
F=\widetilde{F}-\nabla \psi, \quad \psi \in W^{1}\left(\mathbb{R}^{3}\right) / \mathbb{R}, \quad \widetilde{F} \in L^{2}\left(\mathbb{R}^{3}\right)^{3}, \quad \operatorname{div}\left(\epsilon_{r} \widetilde{F}\right)=0 .
$$

The function $\psi$ is called the scalar potential associated with $F$ and $\widetilde{F}$ the divergence free part (or rotational part) of $F$, which is usually associated, in electromagnetism, to a vector potential [15,16]. Moreover the linear map $F \mapsto(\widetilde{F}, \nabla \psi)$ is continuous from $L^{2}\left(\mathbb{R}^{3}\right)^{3}$ into $L^{2}\left(\mathbb{R}^{3}\right)^{3} \times W^{1}\left(\mathbb{R}^{3}\right) / \mathbb{R}$

According to Proposition 3.1, we can decompose $E_{r}^{\delta}$ as (the time regularity of $\widetilde{E}_{r}^{\delta}$ and $\varphi_{r}^{\delta}$ is inherited from the one of $E_{r}^{\delta}$ )

$$
E_{r}^{\delta}=\widetilde{E}_{r}^{\delta}-\nabla \varphi_{r}^{\delta}, \quad \varphi_{r}^{\delta} \in C^{1}\left(\mathbb{R}^{+} ; W^{1}\left(\mathbb{R}^{3}\right) / \mathbb{R}\right), \quad \widetilde{E}_{r}^{\delta} \in C^{1}\left(\mathbb{R}^{+} ; L^{2}\left(\mathbb{R}^{3}\right)^{3}\right), \quad \operatorname{div}\left(\epsilon_{r} \widetilde{E}_{r}^{\delta}\right)=0 .
$$

Thanks to Theorems 3.1 and 3.2 we know that solutions of problem (3.52) and (3.55) exists and are unique, a more precise version of $(3.58)$ is

$$
\left(-\nabla \varphi_{r}^{\delta}, u_{r}^{\delta}, \tilde{E}_{r}^{\delta}\right) \quad \longrightarrow \quad\left(-\nabla \varphi_{r}, u_{r}, 0\right) \quad \text { when } \delta \longrightarrow 0
$$

We can now state our main theorem.

Theorem 3.3. Let $\left(E_{r}^{\delta}, H_{r}^{\delta}, u_{r}^{\delta}\right)$ be the solution of the problem (3.52) with initial conditions satisfying (3.59)-(3.62) with source terms satisfying (3.61)-(3.63) and let $\left(\nabla \varphi_{r}, u_{r}\right)$ be the solution of the limit problem (3.55) with the same initial condition and sources then there exists a function $M_{0}(t)>0 \in C^{0}\left(\mathbb{R}^{+}\right)$, depending only on $\left(\gamma, \epsilon_{r}, \mu_{r}, \mathbf{d}_{r}, \mathbf{C}_{r}, \rho_{r}, \varphi_{r}^{0}, u_{r}^{0}, u_{r}^{1}, \jmath_{r}, m_{r}, f_{r}\right)$ such that $\forall t>0$

$$
\left\|\left(\varphi_{r}^{\delta}-\varphi_{r}\right)(t)\right\|_{\mathbf{W}}+\left\|\frac{\partial}{\partial t}\left(u_{r}^{\delta}-u_{r}\right)(t)\right\|_{L^{2}\left(\widehat{\Omega}_{S}\right)}+\left\|e\left(u_{r}^{\delta}-u_{r}\right)(t)\right\|_{L^{2}\left(\widehat{\Omega}_{S}\right)} \leq \delta M_{0}(t),
$$

where we use $\epsilon_{r}$ for the norm on $\mathbf{W}$. Moreover there also exists $M_{1}(t)>0 \in C^{0}\left(\mathbb{R}^{+}\right)$depending on the same parameters as $M_{0}(t)$ such that $\forall t>0$

$$
\left\|\tilde{E}_{r}^{\delta}\right\|_{L^{2}\left(\widehat{\Omega}_{S}\right)} \leq \delta M_{1}(t)
$$

Proof. Let us first indicate the main ideas of the proof

(1) One first establishes that $\left(\varphi_{r}^{\delta}-\varphi_{r}, u_{r}^{\delta}-u_{r}\right)$ satisfies a "quasistatic piezoelectric problem" where $\widetilde{E}_{r}^{\delta}$ plays the role of the (only) source term;

(2) from standard energy analysis for the quasistatic problem, one estimates $\varphi_{r}^{\delta}-\varphi_{r}$ and $u_{r}^{\delta}-u_{r}$, in appropriate norms, with respect to $\widetilde{E}_{r}^{\delta}$

(3) from standard energy analysis for the original problem, one obtains, using a Poincaré-Friedrichs inequality, the desired a priori estimates for $\widetilde{E}_{r}^{\delta}$ as a function of $\delta$. 
Step 1. We take the divergence of the second equation of (3.52) and integrate the result in time between 0 and $t$. Next, using (3.66), we see that $\left(\varphi_{r}^{\delta}, u_{r}^{\delta}\right)$ satisfies:

$$
\begin{cases}\operatorname{div}\left(\epsilon_{r} \nabla \varphi_{r}^{\delta}\right)-\gamma \operatorname{div}\left(\mathbf{d}_{r}^{T} e\left(u_{r}^{\delta}\right)\right)=\operatorname{div}\left(J_{r}+\epsilon E_{r}^{0}-\gamma \mathbf{d}_{r}^{T} e\left(u_{r}^{0}\right)\right), & x \in \mathbb{R}^{3}, \quad t>0 \\ \rho_{r} \frac{\partial^{2}}{\partial t^{2}} u_{r}^{\delta}-\operatorname{div} \mathbf{C}_{r} e\left(u_{r}^{\delta}\right)-\gamma \operatorname{div} \mathbf{d}_{r} \nabla \varphi_{r}^{\delta}=f_{r}-\operatorname{div} \mathbf{d}_{r} \widetilde{E}_{r}^{\delta}, & x \in \widehat{\Omega}_{S}, \quad t>0\end{cases}
$$

as well as the boundary condition

$$
\left(\mathbf{C}_{r} e\left(u_{r}^{\delta}\right)-\left(\mathbf{d}_{r} \nabla \varphi_{r}^{\delta}\right)\right) \mathbf{n}=-\left(\mathbf{d}_{r} \widetilde{E}_{r}^{\delta}\right) \mathbf{n} \quad x \in \partial \Omega_{S} .
$$

By subtracting (3.70) and (3.55), we see that $\left(\varphi_{r}^{\delta}-\varphi_{r}, u_{r}^{\delta}-u_{r}\right)$ satisfies

$$
\begin{cases}\operatorname{div}\left(\epsilon_{r} \nabla\left(\varphi_{r}^{\delta}-\varphi_{r}\right)\right)-\gamma \operatorname{div}\left(\mathbf{d}_{r}^{T} e\left(u_{r}^{\delta}-u_{r}\right)\right)=0, & x \in \mathbb{R}^{3}, \quad t>0, \\ \rho_{r} \frac{\partial^{2}}{\partial t^{2}}\left(u_{r}^{\delta}-u_{r}\right)-\operatorname{div} C_{r} e\left(u_{r}^{\delta}-u_{r}\right)-\gamma \operatorname{div} \mathbf{d}_{r} \nabla\left(\varphi_{r}^{\delta}-\varphi_{r}\right)=-\operatorname{div} \mathbf{d}_{r} \widetilde{E}_{r}^{\delta}, & x \in \widehat{\Omega}_{S}, \quad t>0,\end{cases}
$$

with the boundary condition (by subtracting (3.71) and (3.57))

$$
\left(\mathbf{C}_{r} e\left(u_{r}^{\delta}-u_{r}\right)-\left(\mathbf{d}_{r} \nabla\left(\varphi_{r}^{\delta}-\varphi_{r}\right)\right)\right) \mathbf{n}=-\left(\mathbf{d}_{r} \widetilde{E}_{r}^{\delta}\right) \mathbf{n} \quad x \in \partial \widehat{\Omega}_{S}
$$

and zero initial data

$$
\left(u_{r}^{\delta}-u_{r}\right)(x, 0)=\frac{\partial}{\partial t}\left(u_{r}^{\delta}-u_{r}\right)(x, 0)=0, \quad x \in \widehat{\Omega}_{s} .
$$

Step 2. Introducing the energy

$$
\mathcal{E}_{q s}^{\delta}(t)=\frac{1}{2} \int_{\mathbb{R}^{3}}\left|\epsilon_{r}^{\frac{1}{2}} \nabla\left(\varphi_{r}^{\delta}-\varphi_{r}\right)\right|^{2} \mathrm{~d} x+\frac{1}{2} \int_{\widehat{\Omega}_{S}}\left(\rho_{r}\left|\frac{\partial}{\partial t}\left(u_{r}^{\delta}-u_{r}\right)\right|^{2}+\mathbf{C}_{r} e\left(u_{r}^{\delta}-u_{r}\right): e\left(u_{r}^{\delta}-u_{r}\right)\right) \mathrm{d} x
$$

we obtain easily from (3.72) and (3.73) the identity

$$
\frac{\mathrm{d}}{\mathrm{d} t} \mathcal{E}_{q s}^{\delta}(t)=\int_{\widehat{\Omega}_{S}} \mathbf{d}_{r} \widetilde{E}_{r}^{\delta}: \frac{\partial}{\partial t} e\left(u_{r}^{\delta}-u_{r}\right) \mathrm{d} x
$$

We integrate (3.76) in time between 0 and $t$ to obtain, after integration by parts on the right hand side

$$
\mathcal{E}_{q s}^{\delta}(t)=-\int_{0}^{t} \int_{\widehat{\Omega}_{S}} \mathbf{d}_{r} \frac{\partial}{\partial t} \widetilde{E}_{r}^{\delta}: e\left(u_{r}^{\delta}-u_{r}\right) \mathrm{d} x \mathrm{~d} s+\int_{\widehat{\Omega}_{S}} \mathbf{d}_{r} \widetilde{E}_{r}^{\delta}: e\left(u_{r}^{\delta}-u_{r}\right) \mathrm{d} x
$$

In what follows, $C$ will be the generic notation for a positive constant which depend only on the upper and lower bounds relative to $\epsilon_{r}, \mu_{c}, \rho_{r}, \mathbf{C}_{r}$ and $\mathbf{d}_{r}$ but do not depend on $\delta$. As usual, the value of $C$ may vary from one line to the other.

From the properties of $\mathbf{d}_{r}$ and $\mathbf{C}_{r}$, we deduce that

$$
\begin{aligned}
& \left|\mathbf{d}_{r} \widetilde{E}_{r}^{\delta}: e\left(u_{r}^{\delta}-u_{r}\right)\right| \leq C\left|\widetilde{E}_{r}^{\delta}\right|\left|\mathbf{C}_{r} e\left(u_{r}^{\delta}-u_{r}\right): e\left(u_{r}^{\delta}-u_{r}\right)\right|^{\frac{1}{2}}, \\
& \left|\frac{\partial}{\partial t} \mathbf{d}_{r} \widetilde{E}_{r}^{\delta}: e\left(u_{r}^{\delta}-u_{r}\right)\right| \leq C\left|\frac{\partial}{\partial t} \widetilde{E}_{r}^{\delta}\right|\left|\mathbf{C} e\left(u_{r}^{\delta}-u_{r}\right): e\left(u_{r}^{\delta}-u_{r}\right)\right|^{\frac{1}{2}},
\end{aligned}
$$


which implies, thanks to Cauchy-Schwarz inequality, that

$$
\begin{aligned}
& \left|\int_{\widehat{\Omega}_{S}} \mathbf{d}_{r} \widetilde{E}_{r}^{\delta}: e\left(u_{r}^{\delta}-u_{r}\right) \mathrm{d} x\right| \leq C\left\|\widetilde{E}_{r}^{\delta}(\cdot, t)\right\|_{L^{2}\left(\hat{\Omega}_{S}\right)} \mathcal{E}_{q s}^{\delta}(t)^{\frac{1}{2}} \\
& \left|\int_{0}^{t} \int_{\widehat{\Omega}_{S}} \mathbf{d}_{r} \frac{\partial}{\partial t} \widetilde{E}_{r}^{\delta}: e\left(u_{r}^{\delta}-u_{r}\right) \mathrm{d} x \mathrm{~d} s\right| \leq C \int_{0}^{t}\left\|\frac{\partial}{\partial t} \widetilde{E}_{r}^{\delta}(\cdot, s)\right\|_{L^{2}\left(\widehat{\Omega}_{S}\right)} \mathcal{E}_{q s}^{\delta}(s)^{\frac{1}{2}} \mathrm{~d} s
\end{aligned}
$$

which, combined to (3.77) and Young's inequality, leads to the a priori estimate

$$
\mathcal{E}_{q s}^{\delta}(t) \leq C\left(\left\|\widetilde{E}_{r}^{\delta}(\cdot, t)\right\|_{L^{2}\left(\hat{\Omega}_{S}\right)}^{2}+\int_{0}^{t}\left\|\frac{\partial}{\partial t} \widetilde{E}_{r}^{\delta}(\cdot, s)\right\|_{L^{2}\left(\hat{\Omega}_{S}\right)} \mathcal{E}_{q s}^{\delta}(s)^{\frac{1}{2}} \mathrm{~d} s\right) .
$$

Finally Gronwall's lemma yields

$$
\mathcal{E}_{q s}^{\delta}(t)^{\frac{1}{2}} \leq C\left(\left\|\widetilde{E}_{r}^{\delta}(\cdot, t)\right\|_{L^{2}\left(\hat{\Omega}_{S}\right)}+\int_{0}^{t}\left\|\frac{\partial}{\partial t} \widetilde{E}_{r}^{\delta}(\cdot, s)\right\|_{L^{2}\left(\hat{\Omega}_{S}\right)} \mathrm{d} s\right) .
$$

Step 3. Notice that

$$
\left(\dot{E}_{r}^{\delta}, \dot{H}_{r}^{\delta}, \dot{u}_{r}^{\delta}\right):=\left(\frac{\partial}{\partial t} E_{r}^{\delta}, \frac{\partial}{\partial t} H_{r}^{\delta}, \frac{\partial}{\partial t} u_{r}^{\delta}\right)
$$

satisfies the same problem $(3.52)$ as $\left(E_{r}^{\delta}, H_{r}^{\delta}, u_{r}^{\delta}\right)$ with source terms

$$
\left(j_{r}, \dot{m}_{r}, \dot{f}_{r}\right):=\left(\frac{\partial}{\partial t} \jmath_{r}, \frac{\partial}{\partial t} m_{r}, \frac{\partial}{\partial t} f_{r}\right)
$$

with $\delta$-independent zero initial data (this is where we need well prepared initial data), using (3.59) and (3.60), we denote

$$
\begin{cases}\dot{E}_{r}^{\delta}(\cdot, 0)=\epsilon_{r}^{-1}\left(\gamma \mathbf{d}_{r} e\left(u^{1}\right)+\jmath_{r}(\cdot, 0)\right) & :=\dot{E}_{r}^{0} \\ \dot{H}_{r}^{\delta}(\cdot, 0)=\mu_{r}^{-1} m_{r}(\cdot, 0) & :=\dot{H}_{r}^{0} \\ \dot{u}_{r}^{\delta}(\cdot, 0)=u_{r}^{1} & :=\dot{u}_{r}^{0} \\ \frac{\partial}{\partial t} \dot{u}_{r}^{\delta}(\cdot, 0)=\rho_{r}^{-1}\left(\operatorname{div}\left[\mathbf{C}_{r} e\left(u_{r}^{0}\right)-\gamma \mathbf{d}_{r} \nabla \varphi_{r}^{0}\right]\right) & :=\dot{u}_{r}^{1} .\end{cases}
$$

Remark 3.4. If (3.59) and (3.60) did not hold, an additional term would appear in the right hand side of each of the first two equations of (3.82), namely $\delta^{-1} \nabla \times H_{r}^{0}$ and $\delta^{-1} \nabla \times E_{r}^{0}$. Since these terms are proportional to $1 / \delta$ we need them to be 0 in order to get uniform estimates. This explains (3.59) and (3.60).

Therefore, introducing the "first order" energy

$$
\dot{\mathcal{E}}^{\delta}(t)=\frac{1}{2} \int_{\mathbb{R}^{3}}\left(\left|\epsilon_{r}^{\frac{1}{2}} \dot{E}_{r}^{\delta}\right|^{2}+\left|\mu_{r}^{\frac{1}{2}} \dot{H}_{r}^{\delta}\right|^{2}\right) \mathrm{d} x+\frac{1}{2} \int_{\Omega_{S}}\left(\rho_{r}\left|\frac{\partial}{\partial t} \dot{u}_{r}^{\delta}\right|^{2}+\mathbf{C}_{r} e\left(\dot{u}_{r}^{\delta}\right): e\left(\dot{u}_{r}^{\delta}\right)\right) \mathrm{d} x
$$

and according to (3.16), we have

$$
\dot{\mathcal{E}}^{\delta}(t)^{\frac{1}{2}} \leq \dot{\mathcal{E}}_{+}(t)^{\frac{1}{2}}
$$


where by definition

$$
\dot{\mathcal{E}}_{+}(t)^{\frac{1}{2}}:=\dot{\mathcal{E}}(0)^{\frac{1}{2}}+\int_{0}^{t}\left(\int_{\mathbb{R}^{3}}\left|\epsilon^{-\frac{1}{2}} j_{r}(\cdot, s)\right|^{2}+\left|\mu_{r}^{-\frac{1}{2}} \dot{m}_{r}(\cdot, s)\right|^{2} \mathrm{~d} x+\int_{\Omega_{S}} \rho_{r}^{-1}\left|\dot{f}_{r}(\cdot, s)\right|^{2} \mathrm{~d} x\right)^{\frac{1}{2}} \mathrm{~d} s
$$

and

$$
\dot{\mathcal{E}}(0)=\frac{1}{2} \int_{\mathbb{R}^{3}}\left(\left|\epsilon_{r}^{\frac{1}{2}} \dot{E}_{r}^{0}\right|^{2}+\left|\mu_{r}^{\frac{1}{2}} \dot{H}_{r}^{0}\right|^{2}\right) \mathrm{d} x+\frac{1}{2} \int_{\widehat{\Omega}_{S}}\left(\rho_{r}\left|\dot{u}_{r}^{1}\right|^{2}+\mathbf{C}_{r} e\left(\dot{u}_{r}^{0}\right): e\left(\dot{u}_{r}^{0}\right)\right) \mathrm{d} x .
$$

Next we remark that, from the first equation of (3.52)

$$
\left\|\nabla \times E_{r}^{\delta}(\cdot, t)\right\|_{L^{2}\left(\mathbb{R}^{3}\right)} \leq C \delta\left(\left\|\frac{\partial}{\partial t} H_{r}^{\delta}(\cdot, t)\right\|_{L^{2}\left(\mathbb{R}^{3}\right)}+\left\|m_{r}(., t)\right\|_{L^{2}\left(\mathbb{R}^{3}\right)}\right) \leq C \delta\left(\dot{\mathcal{E}}^{\delta}(t)^{\frac{1}{2}}+\left\|m_{r}(., t)\right\|_{L^{2}\left(\mathbb{R}^{3}\right)}\right),
$$

to deduce that

$$
\left\|\nabla \times E_{r}^{\delta}(\cdot, t)\right\|_{L^{2}\left(\mathbb{R}^{3}\right)} \leq C \delta\left(\dot{\mathcal{E}}_{+}(t)^{\frac{1}{2}}+\left\|m_{r}(., t)\right\|_{L^{2}\left(\mathbb{R}^{3}\right)}\right) .
$$

Next we use a Poincaré-Friedrichs like inequality. Let us introduce the Hilbert space

$$
\mathcal{W}_{\epsilon_{r}}\left(\mathbb{R}^{3}\right)=\left\{v \in L^{2}\left(\mathbb{R}^{3}\right)^{3} / \frac{v}{\sqrt{1+|x|^{2}}} \in L^{2}\left(\mathbb{R}^{3}\right)^{3} \text { such that } \nabla \times v \in L^{2}\left(\mathbb{R}^{3}\right)^{3} \text { and } \nabla \cdot \epsilon_{r} v=0\right\},
$$

equipped with the norm

$$
\|v\|_{W_{\epsilon_{r}}\left(\mathbb{R}^{3}\right)}^{2}=\left\|\frac{v}{\sqrt{1+|\mathbf{x}|^{2}}}\right\|_{L^{2}\left(\mathbb{R}^{3}\right)}^{2}+\|\nabla \times v\|_{L^{2}\left(\mathbb{R}^{3}\right)}^{2} .
$$

The following result is proved in appendix:

Theorem 3.4. Assume that $\epsilon_{r}$ is constant outside the ball centered at zero and radius $R$ (for some $R>0$ ). Then, there exists a constant $C>0$ such that:

$$
\forall v \in \mathcal{W}_{\epsilon_{r}}\left(\mathbb{R}^{3}\right), \quad\left\|\frac{v}{\sqrt{1+|\mathbf{x}|^{2}}}\right\|_{L^{2}\left(\mathbb{R}^{3}\right)} \leq C\|\nabla \times v\|_{L^{2}\left(\mathbb{R}^{3}\right)} .
$$

As a direct consequence of Theorem 3.4, we deduce that, since $\widehat{\Omega}_{S}$ is bounded, there exists a constant $C\left(\widehat{\Omega}_{S}\right)>0$ such that

$$
\forall v \in \mathcal{W}_{\epsilon_{r}}\left(\mathbb{R}^{3}\right), \quad\|v\|_{L^{2}\left(\widehat{\Omega}_{S}\right)} \leq C\left(\widehat{\Omega}_{S}\right)\|\nabla \times v\|_{L^{2}\left(\mathbb{R}^{3}\right)} .
$$

Applying this inequality to $\widetilde{E}_{r}^{\delta}(\cdot, t)$, which belongs to $\mathcal{W}_{\epsilon_{r}}\left(\mathbb{R}^{3}\right)$ and satisfies $\nabla \times \widetilde{E}_{r}^{\delta}=\nabla \times E_{r}^{\delta}$, we deduce from (3.86) that

$$
\left\|\widetilde{E}_{r}^{\delta}(\cdot, t)\right\|_{L^{2}\left(\widehat{\Omega}_{S}\right)} \leq C \delta\left(\dot{\mathcal{E}}_{+}(t)^{\frac{1}{2}}+\left\|m_{r}(., t)\right\|_{L^{2}\left(\mathbb{R}^{3}\right)}\right),
$$

which gives the value for $M_{1}(t)$ in estimate (3.69)

$$
M_{1}(t)=C\left(\dot{\mathcal{E}}_{+}(t)^{\frac{1}{2}}+\left\|m_{r}(., t)\right\|_{L^{2}\left(\mathbb{R}^{3}\right)}\right) .
$$

Next we repeat the argument with:

$$
\left(\ddot{E}_{r}^{\delta}, \ddot{H}_{r}^{\delta}, \ddot{u}_{r}^{\delta}\right):=\left(\frac{\partial^{2}}{\partial t^{2}} E_{r}^{\delta}, \frac{\partial^{2}}{\partial t^{2}} H_{r}^{\delta}, \frac{\partial^{2}}{\partial t^{2}} u_{r}^{\delta}\right),
$$


that satisfies the same problem $(3.52)$ as $\left(E_{r}^{\delta}, H_{r}^{\delta}, u_{r}^{\delta}\right)$ with source terms

$$
\left(\ddot{\jmath}_{r}, \ddot{m}_{r}, \ddot{f}_{r}\right):=\left(\frac{\partial^{2}}{\partial t^{2}} \jmath_{r}, \frac{\partial^{2}}{\partial t^{2}} m_{r}, \frac{\partial^{2}}{\partial t^{2}} f_{r}\right)
$$

with $\delta$-independent zero initial (thanks to the conditions (3.62)) defined by

$$
\begin{cases}\ddot{E}_{r}^{\delta}(\cdot, 0)=\epsilon_{r}^{-1} \frac{\partial}{\partial t} \jmath_{r}(\cdot, 0)-\gamma \epsilon_{r}^{-1} \mathbf{d}_{r}^{T} e\left[\rho_{r}^{-1} f(\cdot, 0)-\rho_{r}^{-1} \operatorname{div}\left(\mathbf{C}_{r} e\left(u^{0}\right)-\gamma \mathbf{d}_{r} \nabla \varphi_{r}^{0}\right)\right] & :=\ddot{E}_{r}^{0}, \\ \ddot{H}_{r}^{\delta}(\cdot, 0)=\mu_{r}^{-1} \frac{\partial}{\partial t} m_{r}(\cdot, 0) & :=\ddot{H}_{r}^{0}, \\ \ddot{u}_{r}^{\delta}(\cdot, 0)=\rho_{r}^{-1}\left(\operatorname{div}\left[\mathbf{C}_{r} e\left(u_{r}^{0}\right)-\gamma \mathbf{d}_{r} \nabla \varphi_{r}^{0}\right]\right) & :=\ddot{u}_{r}^{0}, \\ \frac{\partial}{\partial t} \ddot{u}_{r}^{\delta}(\cdot, 0)=\rho_{r}^{-1}\left(\operatorname{div}\left[\mathbf{C}_{r} e\left(u_{r}^{1}\right)+\gamma^{2} \mathbf{d}_{r}^{T} \epsilon_{r}^{-1} \mathbf{d}_{r} e\left(u_{r}^{1}\right)-\gamma \mathbf{d}_{r}^{T} \epsilon_{r}^{-1} \jmath_{r}(\cdot, 0)\right]\right) & :=\ddot{u}_{r}^{1} .\end{cases}
$$

Introducing the "second order" energy

$$
\ddot{\mathcal{E}}^{\delta}(t)=\frac{1}{2} \int_{\mathbb{R}^{3}}\left(\epsilon_{r}\left|\ddot{E}_{r}^{\delta}\right|^{2}+\mu\left|\ddot{H}_{r}^{\delta}\right|^{2}\right) \mathrm{d} x+\frac{1}{2} \int_{\Omega_{S}}\left(\rho_{r}\left|\frac{\partial}{\partial t} \ddot{u}_{r}^{\delta}\right|^{2}+\mathbf{C}_{r} e\left(\ddot{u}_{r}^{\delta}\right): e\left(\ddot{u}_{r}^{\delta}\right)\right) \mathrm{d} x
$$

and according to (3.16), we have

$$
\ddot{\mathcal{E}}^{\delta}(t)^{\frac{1}{2}} \leq \ddot{\mathcal{E}}_{+}(t)^{\frac{1}{2}}
$$

where by definition

$$
\ddot{\mathcal{E}}_{+}(t)^{\frac{1}{2}}:=\ddot{\mathcal{E}}(0)^{\frac{1}{2}}+\int_{0}^{t}\left(\int_{\mathbb{R}^{3}}\left|\epsilon^{-\frac{1}{2}} \ddot{j}_{r}(\cdot, s)\right|^{2}+\left|\mu_{r}^{-\frac{1}{2}} \ddot{m}_{r}(\cdot, s)\right|^{2} \mathrm{~d} x+\int_{\Omega_{S}} \rho_{r}^{-1}\left|\ddot{f}_{r}(\cdot, s)\right|^{2} \mathrm{~d} x\right)^{\frac{1}{2}} \mathrm{~d} s
$$

and

$$
\ddot{\mathcal{E}}(0)=\frac{1}{2} \int_{\mathbb{R}^{3}}\left(\left|\epsilon_{r}^{\frac{1}{2}} \ddot{E}_{r}^{0}\right|^{2}+\left|\mu_{r}^{\frac{1}{2}} \ddot{H}_{r}^{0}\right|^{2}\right) \mathrm{d} x+\frac{1}{2} \int_{\widehat{\Omega}_{S}}\left(\rho_{r}\left|\ddot{u}_{r}^{1}\right|^{2}+\mathbf{C}_{r} e\left(\ddot{u}_{r}^{0}\right): e\left(\ddot{u}_{r}^{0}\right)\right) \mathrm{d} x .
$$

With the same technique used to derive (3.87) we obtain

$$
\left\|\frac{\partial}{\partial t} \widetilde{E}_{r}^{\delta}(\cdot, t)\right\|_{L^{2}\left(\hat{\Omega}_{S}\right)} \leq C \delta\left(\ddot{\mathcal{E}}_{+}(t)^{\frac{1}{2}}+\left\|\dot{m}_{r}(., t)\right\|_{L^{2}\left(\mathbb{R}^{3}\right)}\right) .
$$

Finally (3.79) with (3.87) and (3.94) give

$$
\mathcal{E}_{q s}^{\delta}(t)^{\frac{1}{2}} \leq C \delta\left[\dot{\mathcal{E}}_{+}^{\frac{1}{2}}(t)+\left\|m_{r}(., t)\right\|_{L^{2}\left(\mathbb{R}^{3}\right)}+\int_{0}^{t}\left(\ddot{\mathcal{E}}_{+}^{\frac{1}{2}}(t)+\left\|\dot{m}_{r}(., t)\right\|_{L^{2}\left(\mathbb{R}^{3}\right)}\right) \mathrm{d} s\right],
$$

estimate (3.68) can be obtained by noticing that

$$
\left\|\left(\varphi_{r}^{\delta}-\varphi_{r}\right)(t)\right\|_{\mathbf{W}}+\left\|\frac{\partial}{\partial t}\left(u_{r}^{\delta}-u_{r}\right)(t)\right\|_{L^{2}\left(\widehat{\Omega}_{S}\right)}+\left\|e\left(u_{r}^{\delta}-u_{r}\right)(t)\right\|_{L^{2}\left(\hat{\Omega}_{S}\right)} \leq C \mathcal{E}_{q s}^{\delta}(t)^{\frac{1}{2}}
$$

and setting

$$
M_{0}(t)=C\left[\dot{\mathcal{E}}_{+}^{\frac{1}{2}}(t)+\left\|m_{r}(., t)\right\|_{L^{2}\left(\mathbb{R}^{3}\right)}+\int_{0}^{t}\left(\ddot{\mathcal{E}}_{+}^{\frac{1}{2}}(t)+\left\|\dot{m}_{r}(., t)\right\|_{L^{2}\left(\mathbb{R}^{3}\right)}\right) \mathrm{d} s\right] .
$$

Remark 3.5. It is easy to verify from the expression for $M_{0}(t)$ that if the source terms $\left(J_{r}, m_{r}, f_{r}\right)$ are compactly supported in time, $M_{0}(t)$ increases at most linearly in time

$$
M_{0}(t) \leq C t
$$

The same remark holds for $M_{1}(t)$. 


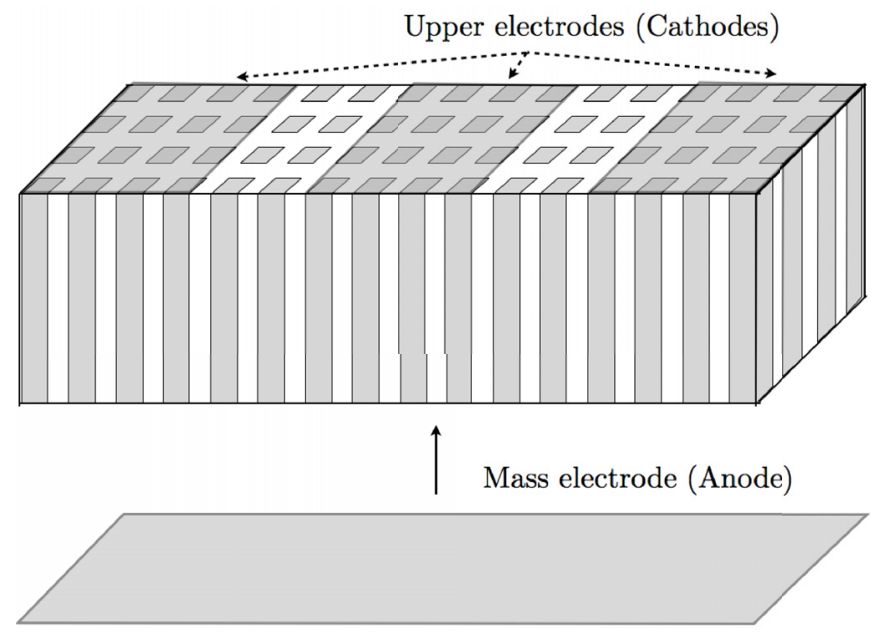

Figure 1. Typical configurations of piezoelectric sensors. Piezoelectric bars are not necessarily in contact with two electrodes.

\section{Modeling OF PIEZOELECTRIC SENSORS}

We now assume a particular geometry for the sensors. More precisely we will study the behavior of sensors made of parallelepipeds bars of piezoelectric material separated by elastic material. The more advanced sensors technology uses piezoelectric bars made of PZT material (see [5]) with an high electric permittivity whereas the elastic material has a low permittivity.

The sensor is connected to several electrodes localized on the top (the cathodes) and the bottom (the anode). For simplicity, these electrodes, which are in practice very thin, will be considered as connected surfaces. Several electrodes can be placed on the top of the sensor whereas a unique electrode is set on the bottom (see Fig. 1). The electrodes are considered as perfect conductors, i.e. we have $E \times \mathbf{n}=\nabla \varphi \times \mathbf{n}=0$ which means, that $\varphi$ is constant in space along each electrode. The anode is grounded at zero potential $(\varphi=0)$.

The section is made of two distinct parts, first we will show how we can use the contrast of permittivities between the piezoelectric bars and the elastic material to reduce the computational domain for the electric potential (which is a priori defined in the whole space) to the piezoelectric elements. Then, we will present boundary conditions that can be used on the cathodes to model the different regimes of the sensor (which are emission or reception regimes) and the corresponding existence and uniqueness theorem that take into account all the previous modeling considerations.

\subsection{Reduction of the computational domain for the electric potential}

We consider the problem (3.22) without electric sources:

$$
\begin{cases}\operatorname{div}(\epsilon \nabla \varphi)-\operatorname{div}\left(\mathbf{d}^{T} e(u)\right)=0, & x \in \mathbb{R}^{3}, \quad t>0, \\ \rho \frac{\partial^{2}}{\partial t^{2}} u-\operatorname{div} \mathbf{C} e(u)-\operatorname{div} \mathbf{d} \nabla \varphi=f, & x \in \Omega_{S}, \quad t>0 .\end{cases}
$$

We do not introduce particular boundary condition for the problem (4.1) and use the stress free condition (3.24) for the displacement variables on the boundary of $\Omega_{S}$ and consider a Laplace equation in the whole space $\mathbb{R}^{3}$ for the electric potential. We assume that the piezoelectric elements consist of an open sub-domain $\Omega_{P} \subset \Omega_{S}$ 
of the elastic domain, as a consequence, outside $\Omega_{P}$ the piezoelectric tensor $\mathbf{d}$ will be zero

$$
\mathbf{d}(x)=0, \quad x \in \Omega_{S} \backslash \bar{\Omega}_{P} .
$$

Realistic designs of sensors include piezoelectric bars with high permittivities surrounded by materials with low permittivities. To be more specific this can be expressed on mathematical terms by

$$
\zeta \ll 1
$$

where $\zeta$ is the dimensionless quantity defined by $(\operatorname{Sp}(\epsilon(x))$ represents the spectrum of the matrix $\epsilon(x))$ :

$$
\zeta=\frac{\epsilon_{\text {ext }}^{+}}{\epsilon_{\text {int }}^{-}}, \quad \epsilon_{\text {ext }}^{+}=\sup _{x \in \mathbb{R}^{3} \backslash \bar{\Omega}_{P}} \max _{\lambda \in \operatorname{Sp}(\epsilon(x))} \lambda, \quad \epsilon_{\mathrm{int}}^{-}=\inf _{x \in \Omega_{P}} \min _{\lambda \in \operatorname{Sp}(\epsilon(x))} \lambda .
$$

We define normalized permittivities $\epsilon_{\text {ext }}^{r}$ for all $x$ in $\mathbb{R}^{3} \backslash \bar{\Omega}_{P}$ and $\epsilon_{\text {int }}^{r}$ for all $x$ in $\Omega_{P}$ such that

$$
\text { a.e. } x \in \mathbb{R}^{3} \backslash \bar{\Omega}_{P}, \quad \epsilon_{\mathrm{ext}}^{r}(x)=\frac{\epsilon(x)}{\epsilon_{\mathrm{ext}}^{+}}, \quad \text { a.e. } x \in \Omega_{P}, \quad \epsilon_{\mathrm{int}}^{r}(x)=\frac{\epsilon(x)}{\epsilon_{\mathrm{int}}^{-}} .
$$

We can rewrite (4.1) as the coupling between elastic equations on $\Omega_{S}$ and a transmission problem between $\Omega_{P}$ and $\mathbb{R}^{3} \backslash \bar{\Omega}_{P}$ for the electric potential where $\varphi$ restricted to $\Omega_{P}\left(\right.$ resp. $\left.\mathbb{R}^{3} \backslash \bar{\Omega}_{P}\right)$ is denoted $\varphi_{\text {int }}\left(\right.$ resp. $\left.\varphi_{\text {ext }}\right)$ :

$$
\left\{\begin{array}{lll}
\operatorname{div}\left(\epsilon_{\mathrm{ext}}^{r} \nabla \varphi_{\mathrm{ext}}\right)=0, & x \in \mathbb{R}^{3} \backslash \bar{\Omega}_{P}, & t>0, \\
\operatorname{div}\left(\epsilon_{\mathrm{int}}^{r} \nabla \varphi_{\mathrm{int}}\right)-\left(\epsilon_{\mathrm{int}}^{-}\right)^{-1} \operatorname{div}\left(\mathbf{d}^{T} e(u)\right)=0, & x \in \Omega_{P}, & t>0, \\
\rho \frac{\partial^{2}}{\partial t^{2}} u-\operatorname{div} \mathbf{C} e(u)-\operatorname{div} \mathbf{d} \nabla \varphi_{\mathrm{int}}=f, & x \in \Omega_{S}, & t>0,
\end{array}\right.
$$

with the following boundary conditions and transmission conditions

$$
\begin{cases}\left(\mathbf{C} e(u)-\mathbf{d} \nabla \varphi_{\mathrm{int}}\right) \cdot \mathbf{n}=0 & x \in \partial \Omega_{S}, \\ \varphi_{\mathrm{int}}=\varphi_{\mathrm{ext}} & x \in \partial \Omega_{P}, \\ \left(\epsilon_{\mathrm{int}}^{r} \nabla \varphi_{\mathrm{int}}-\left(\epsilon_{\mathrm{int}}^{-}\right)^{-1} \mathbf{d}^{T} e(u)\right) \cdot \mathbf{n}=\zeta \epsilon_{\mathrm{ext}}^{r} \nabla \varphi_{\mathrm{ext}} \cdot \mathbf{n} & x \in \partial \Omega_{P} .\end{cases}
$$

The transmission conditions (second and third equation of (4.5) express physically the continuity of the electric potential and the normal electric displacement.

We introduce the extension operator $R_{\text {ext }}$ defined by

$$
\begin{aligned}
R_{\mathrm{ext}}: H^{1 / 2}\left(\Omega_{P}\right) & \longrightarrow W^{1}\left(\mathbb{R}^{3} \backslash \bar{\Omega}_{P}\right) \\
\varphi & \longrightarrow
\end{aligned}
$$

where $\psi$ is the unique solution of the exterior Dirichlet problem (see [16] for instance)

$$
\operatorname{div}\left(\epsilon_{\mathrm{ext}}^{r} \nabla \psi\right)=0, x \in \mathbb{R}^{3} \backslash \bar{\Omega}_{P}, \quad \text { and } \psi=\varphi, \quad x \in \partial \Omega_{P} .
$$

We have the continuity property

$$
\left\|\left(\epsilon_{\mathrm{ext}}^{r}\right)^{\frac{1}{2}} \nabla R_{\mathrm{ext}}(\varphi)\right\|_{L^{2}\left(\mathbb{R}^{3} \backslash \bar{\Omega}_{P}\right)}=\left\|R_{\mathrm{ext}}(\varphi)\right\|_{W^{1}\left(\mathbb{R}^{3} \backslash \bar{\Omega}_{P}\right)} \leq C\|\varphi\|_{H^{1 / 2}\left(\partial \Omega_{P}\right)} .
$$


Using $R_{\text {ext }}$ we define the (non local) Dirichlet to Neumann (DtN) operator $T_{\text {ext }}$ by

$$
\begin{aligned}
& T_{\text {ext }}: H^{1 / 2}\left(\partial \Omega_{P}\right) \longrightarrow H^{-1 / 2}\left(\partial \Omega_{P}\right) \\
& \varphi \quad \longrightarrow-\epsilon_{\mathrm{ext}}^{r} \nabla R_{\mathrm{ext}}(\varphi) \cdot \mathbf{n}
\end{aligned}
$$

where $\mathbf{n}$ is the outward normal of $\Omega_{P}$. It is important to remark that $T_{\text {ext }}$ is a positive operator, indeed

$$
\left\langle T_{\operatorname{ext}}(\varphi), \varphi\right\rangle_{\partial \Omega_{P}}=\int_{\mathbb{R}^{3} \backslash \bar{\Omega}_{P}} \epsilon_{\mathrm{ext}}^{r} \nabla R_{\mathrm{ext}}(\varphi) \cdot \nabla R_{\mathrm{ext}}(\varphi) \mathrm{d} x \geq 0 .
$$

Using $T_{\text {ext }}$, we can reduce the last two equations of (4.5) to a boundary condition for $\varphi_{\text {int }}$ (for simplicity we use the same notation for $\varphi_{\text {int }}$ and its trace on $\left.\partial \Omega_{P}\right)$

$$
\left(\epsilon_{\text {int }}^{r} \nabla \varphi_{\text {int }}-\left(\epsilon_{\text {int }}^{-}\right)^{-1} \mathbf{d}^{T} e(u)\right) \cdot \mathbf{n}=-\zeta T_{\text {ext }}\left(\varphi_{\text {int }}\right) \quad x \in \partial \Omega_{P} .
$$

Using (4.10) we can characterize $\left(u, \varphi_{\text {int }}\right)$ as the solution of a problem on $\Omega_{S} \times \Omega_{P}$ only, the exterior domain being hidden in the DtN operator $T_{\text {ext }}$. To emphasize the dependence in $\zeta$ of the solution and further study the limit behavior when $\zeta$ tends to 0 , we set $\left(u^{\zeta}, \varphi_{\text {int }}^{\zeta}\right) \equiv\left(u, \varphi_{\text {int }}\right)$ which satisfies

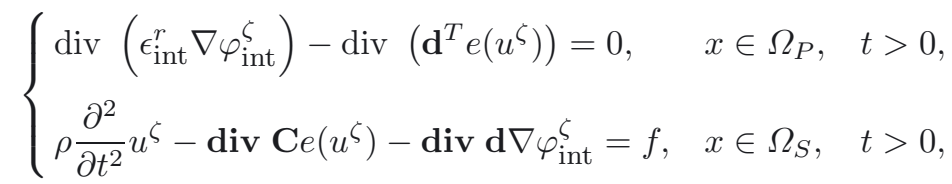

$$
\begin{aligned}
& \left\{\begin{array}{lll}
\left(C e\left(u^{\zeta}\right)+\mathbf{d} \nabla \varphi_{\text {int }}^{\zeta}\right) \cdot \mathbf{n}=0 & x \in \partial \Omega_{S}, & t>0, \\
\left(\epsilon_{\text {int }}^{r} \nabla \varphi_{\text {int }}^{\zeta}-\left(\epsilon_{\text {int }}^{-}\right)^{-1} \mathbf{d}^{T} e\left(u^{\zeta}\right)\right) \cdot \mathbf{n}=-\zeta T_{\text {ext }}\left(\varphi_{\text {int }}^{\zeta}\right) & x \in \partial \Omega_{P} & t>0 .
\end{array}\right.
\end{aligned}
$$

As it has already been emphasized (see also Thm. 3.2), (4.11), (4.12) define only $\varphi_{\text {int }}^{\zeta}$ up to an additive constant.

To completely define $\varphi_{\text {int }}^{\zeta}$ we shall add by convention (which will be useful from the technical point of view)

$$
\int_{\Omega_{P}} \varphi_{\text {int }}^{\zeta} \mathrm{d} x=0
$$

To get rid of the exterior problem, a natural idea is to neglect the " $T_{\text {ext }}$ term" in (4.12), since $\zeta$ is small:

$$
\left(\epsilon_{\mathrm{int}}^{r} \nabla \varphi_{\mathrm{int}}^{\zeta}-\left(\epsilon_{\mathrm{int}}^{-}\right)^{-1} \mathbf{d}^{T} e\left(u^{\zeta}\right)\right) \cdot \mathbf{n}=\zeta T_{\mathrm{ext}}\left(\varphi_{\mathrm{int}}^{\zeta}\right) \rightsquigarrow\left(\epsilon_{\mathrm{int}}^{r} \nabla \varphi_{\mathrm{int}}^{\zeta}-\left(\epsilon_{\mathrm{int}}^{-}\right)^{-1} \mathbf{d}^{T} e\left(u^{\zeta}\right)\right) \cdot \mathbf{n}=0 .
$$

Thus, the problem for the formal limit $\left(u, \varphi_{\text {int }}\right)$ of $\left(u^{\zeta}, \varphi_{\text {int }}^{\zeta}\right)$ when $\zeta$ tends to 0 consists in coupling (4.11) with the boundary conditions

$$
\begin{cases}\left(\mathbf{C} e(u)+\mathbf{d} \nabla \varphi_{\text {int }}\right) \cdot \mathbf{n}=0 & x \in \partial \Omega_{S}, \quad t>0, \\ \left(\epsilon_{\text {int }}^{r} \nabla \varphi_{\text {int }}-\left(\epsilon_{\text {int }}^{-}\right)^{-1} \mathbf{d}^{T} e(u)\right) \cdot \mathbf{n}=0 & x \in \partial \Omega_{P} \quad t>0 .\end{cases}
$$

with the additional condition (deduced from (4.13))

$$
\int_{\Omega_{P}} \varphi_{\text {int }} \mathrm{d} x=0 .
$$

For the rest of the analysis, we need to distinguish two cases. 


\section{The case where $\Omega_{P}$ is a connected domain}

In this case, it is clear that (4.15) uniquely define $\left(u, \varphi_{\text {int }}\right)$ (For conciseness, we omit to state a precise existence and uniqueness result analogous to the one of Theorem 3.2 for $\left(u^{\zeta}, \varphi_{\text {int }}^{\zeta}\right)$. We directly state a convergence theorem with an error estimate.

$$
\left(\varphi_{\text {int }}^{\zeta}, u^{\zeta}\right) \longrightarrow\left(\varphi_{\text {int }}, u\right) \quad \text { when } \quad \zeta \longrightarrow 0
$$

Theorem 4.1. Let $\left(\varphi_{\text {int }}^{\zeta}, u^{\zeta}\right)$ be the solution of the problem (4.11)-(4.13) with data

$$
\left(u_{0}, u_{1}\right) \in H^{1}\left(\Omega_{S}\right)^{3} \times L^{2}\left(\Omega_{S}\right)^{3}, \quad f \in C^{1}\left(\mathbb{R}^{+} ; L^{2}\left(\Omega_{S}\right)^{3}\right),
$$

and let $\left(\varphi_{\text {int }}, u\right)$ be the unique solution of the limit problem (4.11), (4.14), (4.15) with the same initial condition and source term, then there exists a function $M(t)>0 \in C^{0}\left(\mathbb{R}^{+}\right)$, depending only on $\left(\epsilon_{\text {int }}^{r}, \mathbf{d}, \mathbf{C}, \rho, u^{0}, u^{1}, f, \Omega_{P}\right)$ such that for any positive $t$,

$$
\left\|\left(\epsilon_{i n t}^{r}\right)^{\frac{1}{2}} \nabla\left(\varphi_{i n t}^{\zeta}-\varphi_{i n t}\right)(t)\right\|_{L^{2}\left(\Omega_{P}\right)}+\left\|\frac{\partial}{\partial t}\left(u^{\zeta}-u\right)(t)\right\|_{L^{2}\left(\Omega_{S}\right)}+\left\|e\left(u^{\zeta}-u\right)(t)\right\|_{L^{2}\left(\Omega_{S}\right)} \leq \zeta M(t) .
$$

Proof. By substracting (4.11) written with $\left(\varphi_{\text {int }}^{\zeta}, u^{\zeta}\right)$ and $\left(\varphi_{\text {int }}, u\right)$ we obtain the following equations

$$
\begin{cases}\operatorname{div}\left(\epsilon_{\text {int }}^{r} \nabla\left(\varphi_{\text {int }}^{\zeta}-\varphi_{\text {int }}\right)\right)-\operatorname{div}\left(\mathbf{d}^{T} e\left(u^{\zeta}-u\right)\right)=0, & x \in \Omega_{P}, \quad t>0 \\ \rho \frac{\partial^{2}}{\partial t^{2}}\left(u^{\zeta}-u\right)-\operatorname{div} \mathbf{C} e\left(u^{\zeta}-u\right)-\operatorname{div} \mathbf{d} \nabla\left(\varphi_{\text {int }}^{\zeta}-\varphi_{\text {int }}\right)=0, & x \in \Omega_{S}, \quad t>0\end{cases}
$$

with boundary conditions given by subtracting (4.12) with (4.14)

$$
\begin{cases}\left(\mathbf{C} e\left(u^{\zeta}-u\right)+\mathbf{d} \nabla\left(\varphi_{\text {int }}^{\zeta}-\varphi_{\text {int }}\right)\right) \cdot \mathbf{n}=0 & x \in \partial \Omega_{S}, \\ \left(\epsilon_{\text {int }}^{r} \nabla\left(\varphi_{\text {int }}^{\zeta}-\varphi_{\text {int }}\right)-\left(\epsilon_{\text {int }}^{-}\right)^{-1} \mathbf{d}^{T} e\left(u^{\zeta}-u\right)\right) \cdot \mathbf{n}+\zeta T_{\text {ext }}\left(\varphi_{\text {int }}^{\zeta}-\varphi_{\text {int }}\right)=-\zeta T_{\text {ext }}\left(\varphi_{\text {int }}\right) & x \in \partial \Omega_{P} .\end{cases}
$$

Let us introduce the energy

$$
\begin{aligned}
\mathcal{E}_{q s}^{\zeta}(t)= & \frac{1}{2} \int_{\Omega_{P}}\left|\left(\epsilon_{\mathrm{int}}^{r}\right)^{\frac{1}{2}} \nabla\left(\varphi_{\mathrm{int}}^{\zeta}-\varphi_{\mathrm{int}}\right)\right|^{2} \mathrm{~d} x+\frac{1}{2} \int_{\Omega_{S}}\left(\rho\left|\frac{\partial}{\partial t}\left(u^{\zeta}-u\right)\right|^{2}+\mathbf{C} e\left(u^{\zeta}-u\right): e\left(u^{\zeta}-u\right)\right) \mathrm{d} x \\
& +\zeta\left\langle T_{\text {ext }}\left(\varphi_{\mathrm{int}}^{\zeta}-\varphi_{\mathrm{int}}\right), \varphi_{\mathrm{int}}^{\zeta}-\varphi_{\mathrm{int}}\right\rangle_{\partial \Omega_{P}} .
\end{aligned}
$$

By standard techniques (note that the first equation of (4.17) needs to be differentiated in time), we get

$$
\frac{\mathrm{d}}{\mathrm{d} t} \mathcal{E}_{q s}^{\zeta}(t)=-\zeta\left\langle T_{\text {ext }}\left(\frac{\partial}{\partial t} \varphi_{\text {int }}\right), \varphi_{\text {int }}^{\zeta}-\varphi_{\text {int }}\right\rangle_{\partial \Omega_{P}} .
$$

Thus using the trace theorem, Poincaré-Wirtinger's inequality ( $c f .(4.15)$ and (4.13)) and the positivity of $T_{\text {ext }}$ (cf. (4.9)), we get

$$
\frac{\mathrm{d}}{\mathrm{d} t} \mathcal{E}_{q S}^{\zeta}(t) \leq C \zeta\left\|T_{\text {ext }}\left(\frac{\partial}{\partial t} \varphi_{\text {int }}\right)\right\|_{H^{-1 / 2}\left(\Omega_{P}\right)}\left\|\nabla\left(\varphi_{\text {int }}^{\zeta}-\varphi_{\text {int }}\right)\right\|_{L^{2}\left(\Omega_{P}\right)} \leq C \zeta\left\|T_{\text {ext }}\left(\frac{\partial}{\partial t} \varphi_{\text {int }}\right)\right\|_{H^{-1 / 2}\left(\Omega_{P}\right)} \mathcal{E}_{q s}^{\zeta}(t)^{\frac{1}{2}}
$$

One easily concludes using Gronwall's lemma and setting $M(t)=C \int_{0}^{t}\left\|T_{\operatorname{ext}}\left(\frac{\partial}{\partial t} \varphi_{\text {int }}\right)(\cdot, s)\right\|_{H^{-1 / 2}\left(\partial \Omega_{P}\right)} \mathrm{d} s$. 
The decoupling of the limit electric potential between $\Omega_{P}$ and $\mathbb{R}^{3} \backslash \bar{\Omega}_{P}$ does not mean that the exterior electric potential tends to 0 . In fact, the limit exterior potential $\varphi_{\text {ext }}$ can be recovered by "post-processing" as specified in the following corollary, whose proof is trivial since $\varphi_{\text {ext }}^{\zeta}=R_{\text {ext }}\left(\varphi_{\text {int }}^{\zeta}\right)$ :

Corollary 4.1. Let $\varphi_{\text {ext }}=R_{\text {ext }}\left(\varphi_{\text {int }}\right)$, we have

$$
\left\|\left(\varphi_{\text {ext }}^{\zeta}-\varphi_{\text {ext }}\right)(t)\right\|_{W^{1}\left(\mathbb{R}^{3} \backslash \bar{\Omega}_{P}\right)} \leq C \zeta M(t)
$$

\section{The case where $\Omega_{P}$ has several connected components}

We assume that

$$
\Omega_{P}=\bigcup_{j=1}^{J} \Omega_{P}^{j} \quad \text { where the } \Omega_{P}^{j} \text { 's are disjoint connected domains. }
$$

In this case, the situation is slightly more difficult. The subtlety comes from the fact that the condition (4.15) is no longer sufficient to define completely $\varphi_{\text {int }}$ and the construction of the limit $\varphi_{\text {int }}$ of $\varphi_{\text {int }}^{\zeta}$ is more involved. We describe below this construction.

First we define $\varphi_{\text {int }}^{0}$ as the unique solution of (4.11), (4.14) coupled with the $J$ additional constraints

$$
\int_{\Omega_{P}^{j}} \varphi_{\text {int }}^{0} \mathrm{~d} x=0, \quad 1 \leq j \leq J
$$

Next we define $e_{j} \in H^{1 / 2}\left(\partial \Omega_{P}\right)$ such that

$$
\left.e_{j}\right|_{\partial \Omega_{P}^{k}}=\delta_{j k} \quad \text { where } \delta_{j k} \text { is the Kronecker symbol }
$$

and the positive definite $J \times J$ capacity matrix $\mathbf{T}$ defined as

$$
\mathbf{T}_{j k}=\left\langle T_{\text {ext }}\left(e_{j}\right), e_{k}\right\rangle_{\partial \Omega_{P}} .
$$

Let $\mathbf{c}^{0}=\left(\mathbf{c}_{j}^{0}\right) \in \mathbb{R}^{J}$ with $\mathbf{c}_{j}^{0}:=\left\langle T_{\text {ext }}\left(e_{j}\right), \varphi_{\text {int }}^{0}\right\rangle_{\partial \Omega_{P}}$ and $\mathbf{c}=\left(\mathbf{c}_{j}\right) \in \mathbb{R}^{J}$ defined as

$$
\mathbf{c}:=\mathbf{T}^{-1} \mathbf{c}^{0}
$$

we finally define the limit $\varphi_{\text {int }}$ of $\varphi_{\text {int }}^{\zeta}$ as $\left(\chi_{P}^{j}\right.$ is the characteristic function of $\left.\Omega_{P}^{j}\right)$

$$
\varphi_{\text {int }}=\varphi_{\text {int }}^{0}-\sum_{j=1}^{J} \mathbf{c}_{j} \chi_{P}^{j}
$$

It is possible to prove a convergence theorem similar to Theorem 4.1. We omit the details of the proof.

It is important to notice that

- The field $\left(u, \varphi_{\text {int }}^{0}\right)$ can be obtained in practice by solving a problem posed in $\Omega_{S}$ for $u$ and $\Omega_{P}$ for $\varphi_{\text {int }}^{0}$. However, this is not the case for the constants $\mathbf{c}_{j}$ which require the evaluation of operator $T_{\text {int }}^{0}$, i.e. the resolution of exterior problems;

- however, as $\varphi_{\text {int }}$ only differs from $\varphi_{\text {int }}^{0}$ by constant functions inside each $\Omega_{j}$, the corresponding electric fields (the gradients) are the same.

Remark 4.1. As we shall see in the next section, in the presence of electrodes on each $\Omega_{P}^{j}$, the difficulty of "fixing the constants" in each $\Omega_{P}^{j}$ is completely removed because a Dirichlet condition for the electric potential is applied on a non empty part of the boundary of each $\Omega_{P}^{j}$. 

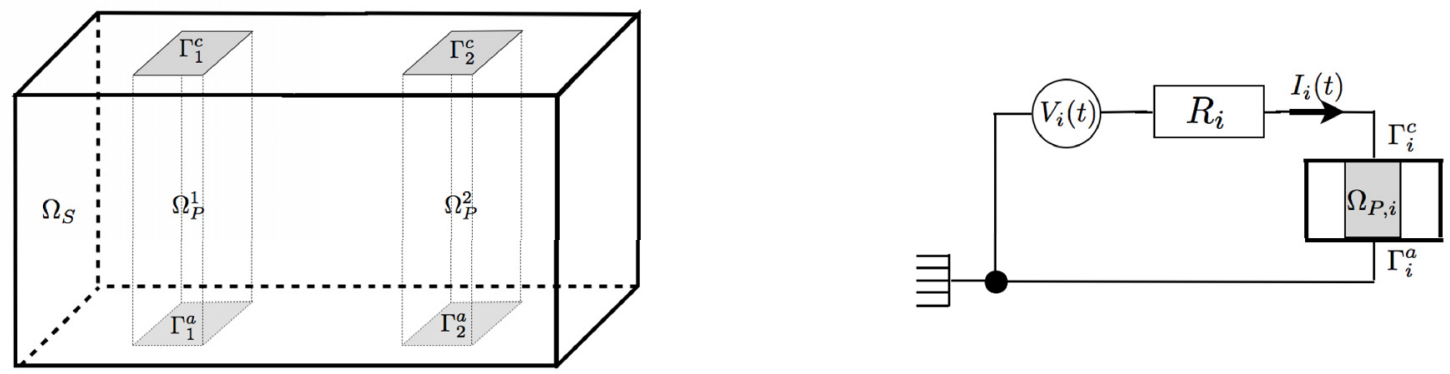

Figure 2. The geometry of a simple sensor (left). Schematic view of the electrical devices (right).

\subsection{Source modeling}

The boundary conditions will enable to model the emission or reception regimes. By the analysis of the previous section, we have shown that we can decouple the electro-static resolution between the bars, we suppose that this remain true in the presence of electrodes (see Fig. 1) but this can be proven rigorously. We now suppose that the piezoelectric domain is composed of piezoelectric bars $\Omega_{P}^{i}$ :

$$
\Omega_{P}=\bigcup_{i=1}^{N_{B}} \Omega_{P}^{i} \quad \text { with } \quad \bar{\Omega}_{P}^{i} \cap \bar{\Omega}_{P}^{j}=\emptyset \text { if } i \neq j .
$$

The boundary of each bar include two surfaces denoted $\Gamma_{i}^{c}$ and $\Gamma_{i}^{a}$ (for cathode and anode):

$$
\partial \Omega_{P, i}=\Gamma_{i}^{c} \cup \Gamma_{i}^{a} \cup \Gamma_{i}^{r} \quad \text { with } \quad \Gamma_{i}^{c} \cap \Gamma_{i}^{a}=\Gamma_{i}^{c} \cap \Gamma_{i}^{r}=\Gamma_{i}^{r} \cap \Gamma_{i}^{a}=\emptyset, \quad \Omega_{P}^{i} \text { connected, } \quad \Gamma_{i}^{a} \neq \emptyset .
$$

See Figure 2 for a description of a standard geometry. Our model reads as

$$
\begin{cases}\operatorname{div}(\epsilon \nabla \varphi)=\operatorname{div}\left(\mathbf{d}^{T} e(u)\right), & x \in \Omega_{P}, \quad t>0, \\ \rho \frac{\partial^{2}}{\partial t^{2}} u-\operatorname{div} \mathbf{C} e(u)=\operatorname{div} \mathbf{d} \nabla \varphi, & x \in \Omega_{S}, \quad t>0,\end{cases}
$$

with the boundary conditions

$$
\begin{aligned}
& (\mathbf{C} e(u)+\mathbf{d} \nabla \varphi) \cdot \mathbf{n}=0, \quad x \in \partial \Omega_{S}, \quad t>0, \\
& \left(\epsilon \nabla \varphi-\mathbf{d}^{T} e(u)\right) \cdot \mathbf{n}=0, \quad x \in \bigcup_{i=1}^{N_{B}} \Gamma_{i}^{r}, \quad t>0 .
\end{aligned}
$$

Boundary conditions are missing for the electric potential, on the cathodes (which corresponds to a particular $\Gamma_{i}^{c}$ ) it will be imposed in such a way that it models the different regimes of the sensor whereas the anode (which corresponds to $\Gamma_{i}^{a}$ ) is always grounded at zero potential, that is, a reference potential is applied, which will be fixed to 0 using the Dirichlet condition $\varphi_{\mid \Gamma_{i}^{a}}=0$.

A particular care must be done when several bars are connected to a same cathode, see Remark 4.2. In the following we consider that each bar is connected to a single cathode.

We are going to use a simplified model for the cathode, one can refer to [18] for a more sophisticated model (that will be the subject of future work) or $[2,10]$ for equivalent approaches . The electric charge on the $i$ th cathode reads

$$
Q_{i}(t)=\int_{\Gamma_{i}^{c}}\left(\epsilon \nabla \varphi-\mathbf{d}^{T} e(u)\right) \cdot \mathbf{n} \mathrm{d} \sigma .
$$




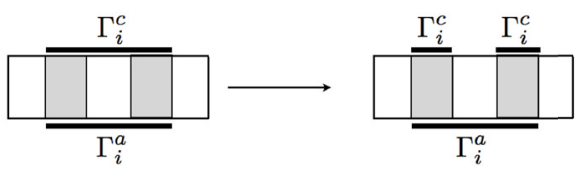

FigurE 3. Splitting hypothesis. The effect of the electrode is neglected between the bars.

We consider that each pair of electrodes (one cathode and one anode) are connected to a generator with an internal resistance (see Fig. 2). Using Ohms' law gives

$$
\varphi_{\mid \Gamma_{i}^{c}}-V_{i}(t)=R_{i} I_{i}(t),
$$

where $I_{i}(t)$ is the electrical current flowing into the piezoelectric bar. As $I_{i}(t)=\mathrm{d} Q_{i}(t) / \mathrm{d} t$ we finally obtain the mixed boundary condition

$$
\varphi_{\mid \Gamma_{i}^{c}}=V_{i}(t)+R_{i} \frac{\mathrm{d}}{\mathrm{d} t} \int_{\Gamma_{i}^{c}}\left(\epsilon \nabla \varphi-\mathbf{d}^{T} e(u)\right) \cdot \mathbf{n} \mathrm{d} \sigma .
$$

By setting $V_{i}(t)=0$, we recover the reception regime, whereas $V(t)$ is a pulse in the emission regime.

Remark 4.2. In the case where severals bars are connected to the same cathode, it is possible to prove, that it is a good approximation to neglect completely the effect of the electrode on the electric field between the bars (see Fig. 3) (this is true only if the permittivities between the bars are small compared to the permittivities of the bars). However one must not forget that the potential on the electrode must be constant all along that electrode even if a mathematical modeling reduction has "broken" the electrode into several parts.

\subsection{Existence-uniqueness result and energy identity}

The full problem can be written

$$
\begin{cases}\operatorname{div}(\epsilon \nabla \varphi)=\operatorname{div}\left(\mathbf{d}^{T} e(u)\right), & x \in \Omega_{P}, \quad t>0, \\ \rho \frac{\partial^{2}}{\partial t^{2}} u-\operatorname{div} \mathbf{C} e(u)=\operatorname{div} \mathbf{d} \nabla \varphi, & x \in \Omega_{S}, \quad t>0,\end{cases}
$$

with the stress free conditions on $\partial \Omega_{S}$

$$
(\mathbf{C} e(u)+\mathbf{d} \nabla \varphi) \mathbf{n}=0, \quad x \in \partial \Omega_{S}, \quad t>0,
$$

and on the boundary of each bars $\left(\partial \Omega_{P, i}\right)$ we use the set of conditions

$$
\begin{cases}\varphi=V_{i}(t)+R_{i} \frac{\mathrm{d}}{\mathrm{d} t} \int_{\Gamma_{i}^{c}}\left(\epsilon \nabla \varphi-\mathbf{d}^{T} e(u)\right) \cdot \mathbf{n} \mathrm{d} \sigma, & x \in \Gamma_{i}^{c}, \quad t>0, \\ \varphi=0 & x \in \Gamma_{i}^{a}, \quad t>0, \\ \left(\epsilon \nabla \varphi-\mathbf{d}^{T} e(u)\right) \cdot \mathbf{n}=0 & x \in \Gamma_{i}^{r}, \quad t>0 .\end{cases}
$$

To complete the problem we need $2+N_{B}$ initial conditions. The first two initial conditions concern the displacement and express that the elastic body is initially at rest

$$
u(x, 0)=\frac{\partial}{\partial t} u(x, 0)=0, \quad x \in \Omega_{S} .
$$


The other conditions express that, on each cathode, the electric charge is:

$$
Q_{i}(0)=\int_{\Gamma_{i}^{c}}\left(\epsilon \nabla \varphi-\mathbf{d}^{T} e(u)\right)(\cdot, 0) \cdot \mathbf{n} \mathrm{d} \sigma=0 .
$$

The first step towards the analysis and numerical approximation (see Sect. 5) of the problem (4.28)-(4.30) is to write the associated variational formulation (or weak formulation in space). To do so we introduce the variational space

$$
\mathcal{X}=H^{1}\left(\Omega_{S}\right)^{3}
$$

corresponding to the elastic variable. Looking at the first equation of (4.30), we see that $\varphi$ is constant on each $\Gamma_{i}^{c}$, which suggest to look for the electric variable in the space

$$
\mathcal{Y}=\left\{\psi \in H^{1}\left(\Omega_{P}\right) \mid \psi=0 \text { on } \Gamma_{i}^{a} \text { and } \psi_{\mid \Gamma_{i}^{c}} \in \mathbb{P}_{0}\left(\Gamma_{i}^{c}\right), \quad 1 \leq i \leq N_{B}\right\} .
$$

It turns out to be useful to decompose $\mathcal{Y}$ as $\mathcal{Y}=\mathcal{Y}_{0} \oplus \mathcal{Y}_{\text {stat }}$ where

$$
\mathcal{Y}_{0}=\left\{\psi \in H^{1}\left(\Omega_{P}\right) \mid \psi=0 \text { on } \Gamma_{i}^{a} \cup \Gamma_{i}^{c} \quad 1 \leq i \leq N_{B}\right\}, \quad \mathcal{Y}_{\text {stat }}=\operatorname{span}\left\{\varphi_{1}, \ldots, \varphi_{N_{B}}\right\}
$$

and each $\varphi_{i}$ is chosen to be the solution of the electro-static problem

$$
\left\{\begin{array} { l l } 
{ \operatorname { d i v } ( \epsilon \nabla \varphi _ { i } ) = 0 , } & { x \in \Omega _ { P } ^ { i } , } \\
{ \varphi _ { i } = 0 } & { x \in \Omega _ { P } \backslash \Omega _ { P } ^ { i } , }
\end{array} \quad \text { and } \quad \left\{\begin{array}{ll}
\varphi_{i}=0, & x \in \Gamma_{i}^{a}, \varphi_{i}=1, x \in \Gamma_{i}^{c}, \\
\epsilon \nabla \varphi_{i} \cdot \mathbf{n}=0 x \in \Gamma_{i}^{r} .
\end{array}\right.\right.
$$

One can remark that if $i \neq j$, we have, since $\varphi_{i}$ and $\varphi_{j}$ have disjoints supports

$$
\int_{\Omega_{P}} \epsilon \nabla \varphi_{i} \cdot \nabla \varphi_{j} \mathrm{~d} x=0
$$

Moreover from (4.33), we also deduce that

$$
\int_{\Omega_{P}} \epsilon \nabla \varphi_{i} \cdot \nabla \varphi_{0} \mathrm{~d} x=\int_{\Gamma_{i}^{c}} \epsilon \nabla \varphi_{i} \cdot \mathbf{n} \varphi_{0} \mathrm{~d} \sigma=0, \quad \forall \varphi_{0} \in \mathcal{Y}_{0}
$$

The decomposition $\mathcal{Y}=\mathcal{Y}_{0} \oplus \mathcal{Y}_{\text {stat }}$ enables us to write

$$
\varphi(x, t)=\varphi_{0}(x, t)+\sum_{i=1}^{N_{B}} \lambda_{i}(t) \varphi_{i}(x), \quad \varphi_{0}(\cdot, t) \text { in } \mathcal{Y}_{0} \text { and } \lambda_{i}(t) \in \mathbb{R} .
$$

It is interesting to emphasize that the scalar function $\lambda_{i}(t)$ is related to the electric charge $Q_{i}(t)(c f .4 .27)$ and the displacement field through the equality (simply substitute (4.36) into (4.27))

$$
Q_{i}(t)+\left(\int_{\Omega_{P}^{i}} \epsilon \nabla \varphi_{i} \cdot \nabla \varphi_{i} \mathrm{~d} x\right) \lambda_{i}(t)-\int_{\Omega_{P}^{i}} \mathbf{d}^{T} e(u(t)) \nabla \varphi_{i} \mathrm{~d} x=0,
$$

After multiplying by test functions, $v$ and $\psi$ in $\mathcal{X}$ and $\mathcal{Y}_{0}$ the two equations of (4.28), we obtain, using the boundary condition (4.29) and the last two equations of (4.30):

$$
\begin{cases}\frac{\mathrm{d}^{2}}{\mathrm{~d} t^{2}} \rho(u(t), v)+a(u(t), v)=-b\left(\varphi_{0}(t), v\right)-\sum_{i=1}^{N_{B}} \lambda_{i}(t) b\left(\varphi_{i}, v\right) & \forall v \in \mathcal{X}, \\ c\left(\varphi_{0}(t), \psi\right)=b(\psi, u(t)) & \forall \psi \in \mathcal{Y}_{0},\end{cases}
$$


where

$$
\begin{aligned}
& \rho(u, v)=\int_{\Omega_{S}} \rho u \cdot v \mathrm{~d} x, \quad \forall(u, v) \in \mathcal{X} \times \mathcal{X}, \quad b(\varphi, u)=\int_{\Omega_{P}} \mathbf{d} \nabla \varphi: e(u) \mathrm{d} x, \forall(\varphi, u) \in \mathcal{Y} \times \mathcal{X}, \\
& a(u, v)=\int_{\Omega_{S}} C e(u): e(v) \mathrm{d} x, \forall(u, v) \in \mathcal{X} \times \mathcal{X}, \quad c(\varphi, \psi)=\int_{\Omega_{P}} \epsilon \nabla \varphi \cdot \nabla \psi \mathrm{d} x, \quad \forall(\varphi, \psi) \in \mathcal{Y} \times \mathcal{Y} .
\end{aligned}
$$

Note that, at this stage, the equation on the $\Gamma_{i}^{c}$ has not been used since the trace on $\Gamma_{i}^{c}$ of test functions in $\mathcal{Y}_{0}$ vanish. We need to deduce new equations by taking the $\varphi_{j}$ 's as test functions for the first equation of (4.28). By differentiating the first equation of (4.28) in time, multiplying by $\varphi_{j}$ and integrating over $\Omega_{P}$, we obtain:

$$
\int_{\Omega_{P}} \epsilon \nabla \frac{\partial}{\partial t} \varphi \cdot \nabla \varphi_{j} \mathrm{~d} x=\int_{\Omega_{P}} \mathbf{d}^{T} e\left(\frac{\partial}{\partial t} u\right) \cdot \nabla \varphi_{j} \mathrm{~d} x-\sum_{i=1}^{N_{B}} \int_{\Gamma_{i}^{c}} \frac{\partial}{\partial t}\left(\varphi-\mathbf{d}^{T} e(u)\right) \cdot \mathbf{n} \varphi_{j} \mathrm{~d} \mathbf{s},
$$

where the homogeneous equation of (4.30) has been used to get ride of the integrals on $\Gamma_{i}^{a}$ and $\Gamma_{i}^{r}$. Now, using that $\varphi_{j}$ equals 1 on $\Gamma_{j}^{c}$, has a support embedded in $\Omega_{P}^{j}$ and the first equation of (4.30) we can write

$$
\sum_{i=1}^{N_{B}} \int_{\Gamma_{i}^{c}} \frac{\partial}{\partial t}\left(\varphi-\mathbf{d}^{T} e(u)\right) \cdot \mathbf{n} \varphi_{j} \mathrm{~d} \sigma=\frac{1}{R_{j}}\left(\varphi_{\mid \Gamma_{j}^{c}}-V_{j}\right),
$$

which enable us, with (4.34) and (4.35), to deduce an ordinary differential equation for each $\lambda_{j}$ (we introduce the impedance $Z_{j}=1 / R_{j}$ )

$$
c\left(\varphi_{j}, \varphi_{j}\right) \frac{\mathrm{d}}{\mathrm{d} t} \lambda_{j}(t)+Z_{j} \lambda_{j}(t)=\frac{\mathrm{d}}{\mathrm{d} t} b\left(\varphi_{j}, u(t)\right)+Z_{j} V_{j}(t) \quad 1 \leq j \leq N_{B},
$$

that we complete by an initial condition that will replace the initial condition on the electric charge (4.32) using (4.37) and (4.31)

$$
\lambda_{j}(0)=0, \quad 1 \leq j \leq N_{B}
$$

The full problem reads:

$$
\begin{cases}\text { Find }\left(u, \varphi_{0}, \Lambda=\left(\lambda_{i}\right)\right): \mathbb{R}^{+} \longrightarrow \mathcal{X} \times \mathcal{Y}_{0} \times \mathbb{R}^{N_{B}} \text { such that } & \\ \frac{\mathrm{d}^{2}}{\mathrm{~d} t^{2}} \rho(u(t), v)+a(u(t), v)=-b\left(\varphi_{0}(t), v\right)-\sum_{i=1}^{N_{B}} b\left(\varphi_{i}, v\right) \lambda_{i}(t) & \forall v \in \mathcal{X}, \\ c\left(\varphi_{0}(t), \psi\right)=b(\psi, u(t)) & \forall \psi \in \mathcal{Y}_{0}, \\ c\left(\varphi_{j}, \varphi_{j}\right) \frac{\mathrm{d}}{\mathrm{d} t} \lambda_{i}(t)+Z_{i} \lambda_{i}(t)=\frac{\mathrm{d}}{\mathrm{d} t} b\left(\varphi_{i}, u(t)\right)+Z_{i} V_{i}(t) & 1 \leq i \leq N_{B},\end{cases}
$$

completed with initial conditions (4.31), (4.41). We easily get an energy identity by choosing as test functions $v=\partial u / \partial t$ and $\psi=\varphi_{0}$ after differentiating in time the second equation of (4.42). After summation, we first get

$$
\frac{1}{2} \frac{\mathrm{d}}{\mathrm{d} t}\left(\rho\left(\frac{\partial}{\partial t} u(t), \frac{\partial}{\partial t} u(t)\right)+a(u(t), u(t))+c\left(\varphi_{0}(t), \varphi_{0}(t)\right)\right)=-\sum_{i=1}^{N_{B}} \lambda_{i}(t) \frac{\mathrm{d}}{\mathrm{d} t} b\left(\varphi_{i}, u(t)\right) .
$$

The right hand side above can be replaced using the third equation of (4.42) multiplied by $\lambda_{i}$, which finally gives

$$
\frac{1}{2} \frac{\mathrm{d}}{\mathrm{d} t} \mathcal{E}(t)+\sum_{i=1}^{N_{B}} Z_{i} \lambda_{i}^{2}(t)=\sum_{i=1}^{N_{B}} Z_{i} V_{i}(t) \lambda_{i}(t)
$$


where the energy $\mathcal{E}(t)$ is defined as

$$
\mathcal{E}(t)=\rho\left(\frac{\partial}{\partial t} u(t), \frac{\partial}{\partial t} u(t)\right)+a(u(t), u(t))+c\left(\varphi_{0}(t), \varphi_{0}(t)\right)+\sum_{i=1}^{N_{B}} c\left(\varphi_{i}, \varphi_{i}\right) \lambda_{i}^{2}(t) .
$$

Using $V_{i}(t) \lambda_{i}(t) \leq \lambda_{i}^{2}(t)+V_{i}^{2}(t) / 4$, we obtain the inequality $\frac{\mathrm{d}}{\mathrm{d} t} \mathcal{E}(t) \leq \frac{1}{4} \sum_{i=1}^{N_{B}} Z_{i} V_{i}^{2}(t)$, which leads to the estimate

$$
\sup _{t \in[0, T]} \mathcal{E}(t) \leq \frac{Z_{i}}{4} \sum_{i=1}^{N_{B}}\left\|V_{i}(t)\right\|_{L^{2}([0, T])}^{2} .
$$

This estimate is the basic estimate to prove the following existence and uniqueness theorem, based on the equivalence between (4.42), (4.31), (4.41) and (4.28), (4.30), (4.31), (4.32):

Theorem 4.2. Assume that $V(t) \in H_{l o c}^{k}\left(\mathbb{R}^{+}\right)$, the problem (4.28), (4.30), (4.31), (4.32) admits a unique solution

$$
u \in C^{k+1}\left(\mathbb{R}^{+} ; L^{2}\left(\Omega_{S}\right)^{3}\right) \cap C^{k}\left(\mathbb{R}^{+} ; H^{1}\left(\Omega_{S}\right)^{3}\right), \quad \varphi \in C^{k}\left(\mathbb{R}^{+} ; H^{1}\left(\Omega_{P}\right)\right) .
$$

\section{NumericAl APPROXIMATION}

We present a numerical method to handle the problem (4.28), (4.30), (4.31), (4.32) via its weak formulation (4.42), (4.31), (4.41). The space discretization relies on a standard Galerkin approach. We use in practice the hexahedral spectral elements (see $[4,6]$ for more details on spectral elements) that provides mass lumping. The time discretization will be done via a particular second order finite difference scheme constructed in such a way that the stability of the fully discrete problem is guaranteed, under a CFL condition, through an energy approach.

\subsection{Spatial discretization}

\subsubsection{Semi discrete problem}

We introduce some finite-dimensional approximation spaces

$$
\mathcal{X}_{h} \subset \mathcal{X}, \quad \text { and } \quad \mathcal{Y}_{0, h} \subset \mathcal{Y}_{0} .
$$

In practice we use the high order spectral elements on hexahedral meshes as defined in [4] which provide good approximation properties (spectral convergence property for instance).

In our presentation, we are going to suppose that the electrostatic functions $\varphi_{i}$ are known. This is the case for instance in the situation where the $\Omega_{P}^{i}$ 's are parallelepipeds, as in figure: $\varphi_{i}$ is simply a " $1 \mathrm{D}$ " linear function that goes from 0 on the anode to 1 on the cathode. If this is not the case, these functions need to be precomputed (which leads to an additional approximation) using again finite elements for instance. The semi-discrete version of $(4.42)$ is

$$
\begin{cases}\text { Find }\left(u_{h}, \varphi_{0, h}, \Lambda_{h}=\left(\lambda_{i, h}\right)\right): \mathbb{R}^{+} \longrightarrow \mathcal{X}_{h} \times \mathcal{Y}_{0, h} \times \mathbb{R}^{N_{B}} \text { such that } & \\ \frac{\mathrm{d}^{2}}{\mathrm{~d} t^{2}} \rho_{h}\left(u_{h}(t), v_{h}\right)+a_{h}\left(u_{h}(t), v_{h}\right)=-b_{h}\left(\varphi_{0, h}(t), v_{h}\right)-\sum_{i=1}^{N_{B}} b_{h}\left(\varphi_{i}, v_{h}\right) \lambda_{i}(t) & \forall v_{h} \in \mathcal{X}_{h}, \\ c_{h}\left(\varphi_{0, h}(t), \psi_{0, h}\right)=b_{h}\left(\psi_{0, h}, u_{h}(t)\right) & \forall \psi_{0, h} \in \mathcal{Y}_{0, h}, \\ c\left(\varphi_{j}, \varphi_{j}\right) \frac{\mathrm{d}}{\mathrm{d} t} \lambda_{i, h}(t)+Z_{i} \lambda_{i, h}(t)=\frac{\mathrm{d}}{\mathrm{d} t} b_{h}\left(\varphi_{i, h}, u_{h}(t)\right)+Z_{i} V_{i}(t) & 1 \leq i \leq N_{B} .\end{cases}
$$


The subscript $h$ in the bilinear forms indicate that they can be computed in an approximate way. In practice, with hexahedral spectral elements, we shall use the Gauss-Lobatto quadrature formulae of appropriate order (see [6] for more details and theory), that provide in particular mass lumping for the elastic unknowns.

We write the algebraic version of the semi discrete problem by introducing the vectors $U_{h}(t)$ and $\Phi_{0, h}(t)$ as the vectors associated to the decomposition of $u_{h}$ and $\varphi_{0, h}$ in appropriate bases $\mathcal{X}_{h}$ and $\mathcal{Y}_{0, h}$ respectively (in practice, these are the standard Lagrange bases).

$$
\left\{\begin{array}{l}
M \frac{\mathrm{d}^{2}}{\mathrm{~d} t^{2}} U_{h}+K U_{h}=-B \Phi_{0, h}-B_{\text {stat }} \Lambda_{h}, \\
C \Phi_{0, h}=B^{T} U_{h}, \\
C_{\text {stat }} \frac{\mathrm{d}}{\mathrm{d} t} \Lambda_{h}+Z_{\text {stat }} \Lambda_{h}=B_{\text {stat }}^{T} \frac{\mathrm{d}}{\mathrm{d} t} U_{h}+Z_{\text {stat }} V_{\text {stat }},
\end{array}\right.
$$

where $M, K, C$ (they depend on $h$ but we dropped the subscript $h$ for simplicity of notation) are square matrices corresponding respectively to the bilinear forms $\rho_{h}(\cdot, \cdot), a_{h}(\cdot, \cdot)$ and $c_{h}(\cdot, \cdot) . C_{\text {stat }}$ and $Z_{\text {stat }}$ are $N_{B} \times N_{B}$ diagonal matrices whose $i$ th element is $c\left(\varphi_{i}, \varphi_{i}\right)$ and $Z_{i}$ (respectively).

Finally $B$ and $B_{\text {stat }}$ are $\operatorname{dim}\left(\mathcal{X}_{h}\right) \times \operatorname{dim}\left(\mathcal{Y}_{0, h}\right)$ and $\operatorname{dim}\left(\mathcal{X}_{h}\right) \times N_{B}$ rectangular matrices associated to the bilinear form $b_{h}(\cdot, \cdot)$ and $V_{\text {stat }}(t)=\left(V_{i}(t)\right) \in \mathbb{R}^{N_{B}}$.

To anticipate the time discretization, we eliminate the vector $\Phi_{0, h}$ which is linked to $U_{h}$ by a static equation. Note that the matrix $C$ is positive definite. We get

$$
\left\{\begin{array}{l}
M \frac{\mathrm{d}^{2}}{\mathrm{~d} t^{2}} U_{h}+\left(K+B C^{-1} B^{T}\right) U_{h}=-B_{\text {stat }} \Lambda_{h} \\
C_{\text {stat }} \frac{\mathrm{d}}{\mathrm{d} t} \Lambda_{h}+Z_{\text {stat }} \Lambda_{h}=B_{\text {stat }}^{T} \frac{\mathrm{d}}{\mathrm{d} t} U_{h}+Z_{\text {stat }} V_{\text {stat }} .
\end{array}\right.
$$

We can prove the stability of the semi-discrete scheme through a semi discrete energy relation that we choose to write in algebraic form. Multiplying (in the sense of inner products) the first equation of (5.3) by $\mathrm{d} U_{h} / \mathrm{d} t$ and the second equation by $\Lambda_{h}$, we obtain after summation $\left((\cdot, \cdot)_{2}\right.$ refers to any euclidian scalar product):

$$
\frac{\mathrm{d}}{\mathrm{d} t} E_{h}(t)+\left(Z_{\text {stat }} \Lambda_{h}, \Lambda_{h}\right)_{2}=\left(Z_{\text {stat }} V_{\text {stat }}, \Lambda_{h}\right)_{2},
$$

with

$$
E_{h}(t)=\left(M \frac{\mathrm{d} U_{h}}{\mathrm{~d} t}, \frac{\mathrm{d} U_{h}}{\mathrm{~d} t}\right)_{2}+\left(\left(K+B C^{-1} B^{T}\right) U_{h}, U_{h}\right)_{2}+\left(C_{\mathrm{stat}} \Lambda_{h}, \Lambda_{h}\right)_{2}
$$

\subsection{Time discretization}

\subsubsection{Presentation of the scheme}

In what follows, we shall use a constant time step $\Delta t$ and for any $F(t)$ we shall denote by $F^{n}$ the approximation of $F\left(t^{n}\right)$ with $t^{n}=n \Delta t$. We look for a second order in time energy preserving scheme, so the natural idea is to user centered approximation of all time derivatives in a leap frog manner. One objective is, in order to limit the computational time, to keep explicit the computation of the displacement field, and at the same time, for robustness, to ensure that the CFL stability condition of the method should not be affected by the modeling of the electric energization process. This led us to consider the following "implicit" approximation for the $\Lambda$ terms in the two equations of (5.3)

$$
\Lambda_{h}\left(t^{n}\right) \simeq \frac{\Lambda_{h}^{n+1}+2 \Lambda_{h}^{n}+\Lambda_{h}^{n-1}}{4} \quad\left(:=\widetilde{\Lambda}_{h}^{n}\right) .
$$

We shall prove in the next section that this choice has has no influence on the CFL condition and we shall see in Section 5.2.3 that the effective computation of $\Lambda_{h}^{n}$ is explicit. 
This leads to the full discrete scheme:

$$
\left\{\begin{array}{l}
M \frac{U_{h}^{n+1}-2 U_{h}^{n}+U_{h}^{n-1}}{\Delta t^{2}}+\left(K+B C^{-1} B^{T}\right) U_{h}^{n}=-B_{\text {stat }} \frac{\Lambda_{h}^{n+1}+2 \Lambda_{h}^{n}+\Lambda_{h}^{n-1}}{4} \\
C_{\text {stat }} \frac{\Lambda_{h}^{n+1}-\Lambda_{h}^{n-1}}{2 \Delta t}+Z_{\text {stat }} \frac{\Lambda_{h}^{n+1}+2 \Lambda_{h}^{n}+\Lambda_{h}^{n-1}}{4}=B_{\text {stat }}^{T} \frac{U_{h}^{n+1}-U_{h}^{n-1}}{2 \Delta t}+Z_{\text {stat }} V_{\text {stat }}^{n} .
\end{array}\right.
$$

\subsubsection{Stability analysis}

We use an energy approach. The first equation of the scheme (5.7) can also be rewritten

$$
\begin{aligned}
\left(M-\frac{\Delta t^{2}}{4}\left(K+B C^{-1} B^{T}\right)\right) \frac{U_{h}^{n+1}-2 U_{h}^{n}+U_{h}^{n-1}}{\Delta t^{2}} \Lambda_{h}+\left(K+B C^{-1} B^{T}\right) & \frac{U_{h}^{n+1}+2 U_{h}^{n}+U_{h}^{n-1}}{4} \\
& =-B_{\text {stat }} \frac{\Lambda_{h}^{n+1}+2 \Lambda_{h}^{n}+\Lambda_{h}^{n-1}}{4} .
\end{aligned}
$$

Multiplying (5.8) by $\frac{U_{h}^{n+1}-U_{h}^{n-1}}{2 \Delta t}$ and (5.7) (ii) by $\widetilde{\Lambda}_{h}^{n}(c f .5 .6)$ gives after summation:

$$
\frac{E^{n+1 / 2}-E^{n-1 / 2}}{2 \Delta t}+\left(Z_{\text {stat }} \widetilde{\Lambda}_{h}^{n}, \widetilde{\Lambda}_{h}^{n}\right)_{2}=\left(Z_{\text {stat }} \widetilde{\Lambda}_{h}^{n}, V_{\text {stat }}^{n}\right)_{2},
$$

where the discrete energy is defined as

$$
\begin{aligned}
E^{n+1 / 2}=( & {\left.\left[M-\frac{\Delta t^{2}}{4}\left(K+B C^{-1} B^{T}\right)\right] \frac{U_{h}^{n+1}-U_{h}^{n}}{\Delta t}, \frac{U_{h}^{n+1}-U_{h}^{n}}{\Delta t}\right)_{2} } \\
& +\left(\left(K+B C^{-1} B^{T}\right) \frac{U_{h}^{n+1}+U_{h}^{n}}{2}, \frac{U_{h}^{n+1}+U_{h}^{n}}{2}\right)_{2}+\frac{1}{2}\left(C_{\text {stat }} \frac{\Lambda_{h}^{n+1}+\Lambda_{h}^{n}}{2}, \frac{\Lambda_{h}^{n+1}+\Lambda_{h}^{n}}{2}\right)_{2} .
\end{aligned}
$$

The identity (5.9) shows, thanks to the positivity of $Z_{\text {stat }}$ that this discrete energy decays when $V_{\text {stat }}^{n}=0$, which corresponds the natural losses in the resistors. More generally, by discrete Gronwall's techniques, one easily deduces from (5.9) a priori bounds for the energy which are independent on the discretization parameters $\Delta t$ and $h$. The stability analysis is reduced to proving the positivity of the energy which obviously amounts to ensuring the positivity of the first term of the right hand side of (5.10). This leads to the CFL sufficient (we conjecture that it is also necessary) time step restriction

$$
\Delta t \leq 2 \rho\left(M^{-1}\left(K+B C^{-1} B^{T}\right)\right)^{-\frac{1}{2}}
$$

where $\rho(A)$ denotes the spectral radius of any matrix $A$. Note that, as announced, this stability condition is independent of $C_{\text {stat }}$ or $Z_{\text {stat }}$, i.e. of the energization process.

\subsubsection{The computational algorithm}

The practical implementation of the scheme (5.7) is done in three steps.

To do so we define $U_{0, h}^{n+1}$ as the solution one would obtain if $\widetilde{\Lambda}_{h}^{n}$ were 0 , namely

$$
M \frac{U_{0, h}^{n+1}-2 U_{h}^{n}+U_{h}^{n-1}}{\Delta t^{2}}+\left(K+B C^{-1} B^{T}\right) U_{h}^{n}=0,
$$

which enables us to rewrite (5.7)(i) as

$$
M U_{h}^{n+1}=M U_{0, h}^{n+1}-\Delta t^{2} B_{\text {stat }} \frac{\Lambda_{h}^{n+1}+2 \Lambda_{h}^{n}+\Lambda_{h}^{n-1}}{4} .
$$


Moreover, eliminating $U_{h}^{n+1}$, using a Schur complement, in (5.7)(ii) leads to

$$
C_{\text {stat }} \frac{\Lambda_{h}^{n+1}-\Lambda_{h}^{n-1}}{2 \Delta t}+\tilde{Z}_{\text {stat }} \frac{\Lambda_{h}^{n+1}+2 \Lambda_{h}^{n}+\Lambda_{h}^{n-1}}{4}=B_{\text {stat }}^{T} \frac{U_{0, h}^{n+1}-U_{h}^{n-1}}{2 \Delta t}+Z_{\text {stat }} V_{\text {stat }}^{n},
$$

with $\tilde{Z}_{\text {stat }}$ defined by

$$
\tilde{Z}_{\text {stat }}=Z_{\text {stat }}+\frac{\Delta t}{2} B_{\text {stat }}^{T} M^{-1} B_{\text {stat }} .
$$

Therefore (5.7) is equivalent to (5.12)-(5.14) and the computational algorithm at time $t^{n+1}$ is

- Prediction. Knowing $U_{h}^{n}$ and $U_{h}^{n-1}$, compute $U_{0, h}^{n+1}$, using (5.12);

- boundary terms. Compute $\Lambda_{h}^{n+1}$ with $U_{0, h}^{n+1}, U_{h}^{n-1}$ and $\Lambda_{h}^{n-1}$ using equation (5.14);

- correction. Compute $U_{h}^{n+1}$ given by (5.13).

The first step of the algorithm implies the inversion of the matrix $C$. In practice we do not store the inverse of the matrix but at each time step we compute $\Phi_{0, h}^{n}$ such that

$$
C \Phi_{0, h}^{n}=B^{T} U_{h}^{n},
$$

this enable to recover the value of the electric potential $\left(\varphi_{h}^{n}\right)$ in the piezoelectric domains (using $\Phi_{0, h}^{n}$ and $\Lambda_{h}^{n}$ in a post-processing process using the decomposition property (4.36)). We emphasize that the the computation of $\Lambda_{h}$ is explicit since $\tilde{Z}_{\text {stat }}$ and $C_{\text {stat }}$ are positive diagonal matrices.

\section{Numerical Results}

Our numerical simulations concern the 2D problem. We look at the configuration described in Figure 4. The physical parameters are given in Table 1. We consider a sensor posed on a isotropic homogeneous half-space with a circular defect (a hole). The sensor is made of twenty piezoelectric bars $\left(N_{B}=20\right)$ separated by a polymer. We use stress free condition on all the elastic boundaries and a perfectly matched layer is used to bound the computational domain.

In Table 1, the density $\rho$ are in $\mathrm{kg} \mathrm{m}^{-3}$, the elastic coefficients $\mathbf{C}_{i j k l}$ are in GPa, the piezoelectric coefficients $\mathbf{d}_{i j}$ in $\mathrm{Cm}^{-1}$ and the permittivities $\epsilon_{i i}$ are given relatively to the permittivity of the vacuum. After taking into account the symmetry properties of the tensors, the remaining coefficients are zero.

We assume that all piezoelectric bars are connected to one cathode and one anode which are linked to a distinct generator whose resistance is set to $R_{i}=1 e^{-3} \Omega$. Each cathode are excited using a source function of the form $V_{i}\left(t ; \tau, f_{0}\right)=-\cos \left(2 \pi f_{0}\left(t-\tau_{i}\right)\right)+1$ when $t \in\left[\tau_{i}, 1 / f_{0}+\tau_{i}\right]$ and 0 otherwise. We choose for all the $V_{i}$, $f_{0}=4 M h z$, the delay $\tau_{i}$ will be set to 0 for all $i$ in a first simulation then in a second simulation the $\tau_{i}$ will depends on the position of the piezoelectric bar it refers to. More precisely, we note $L_{i}$ the distance from the center of the defect to the center of the $i t h$ anode and

$$
L_{+}=\max L_{i}, \quad I=\operatorname{argmax} L_{i},
$$

$V_{+}$will denote the velocity of the pressure wave in the isotropic half-space. We consider that the center of the anode of each piezoelectric bar behave like point sources and we want to generate pressure waves that start from each point sources and reach the center of the defect at the same time, this means that

$$
\frac{L_{i}}{V_{+}}-\tau_{i}=\frac{L_{I}}{V_{+}}-\tau_{I} \quad 1 \leq i \leq N_{B}
$$

setting $\tau_{I}=0$ enable us to compute all the $\tau_{i}$ 's. We recover numerically the potential on the cathodes (given by $\lambda_{i, h}^{n}$ ). Figure 6 represent the potential on the tenth cathode. We clearly see that the focusing effect results in the best detection of the defect. Figures 7 represents different snapshots of the simulation with and without delay. 
TABLE 1. Electric and elastic properties of the materials.

\begin{tabular}{rlrlll}
\hline \multicolumn{6}{c}{ Piezoelectric material } \\
\hline $\mathbf{C}_{1111}$ & 111.0 & $\mathbf{C}_{1122}$ & 15.4 & $\mathbf{C}_{2222}$ & 121.0 \\
$\mathbf{C}_{1212}$ & 21.1 & $\rho$ & 7.75 & & \\
$\mathbf{d}_{112}$ & 12.7 & $\mathbf{d}_{211}$ & -5.4 & $\mathbf{d}_{222}$ & 15.1 \\
$\epsilon_{11}$ & 1730 & $\epsilon_{22}$ & 1700 \\
\hline \multicolumn{5}{c}{ Polymer (isotropic) } \\
\hline $\mathbf{C}_{1111}$ & 8.5 & $\mathbf{C}_{1122}$ & 4.3 & $\rho$ & 1.1 \\
$\epsilon_{11}$ & 4 & $\epsilon_{22}$ & 5 & & \\
\hline \multicolumn{6}{c}{ half-space (isotropic) } \\
\hline $\mathbf{C}_{1111}$ & 27.15 & $\mathbf{C}_{1122}$ & 10.88 & $\rho$ & 7.8 \\
\hline
\end{tabular}

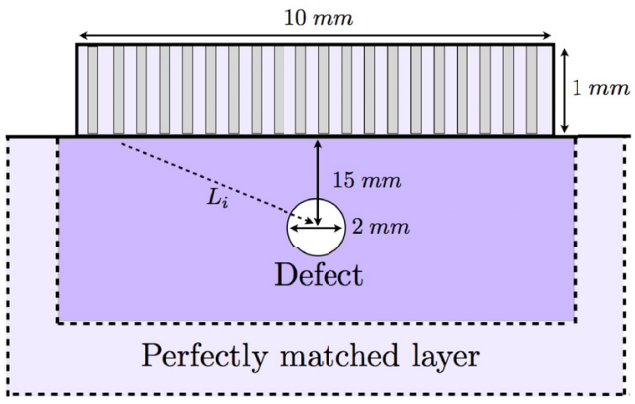

Figure 4. Schematic view of the testing configuration.

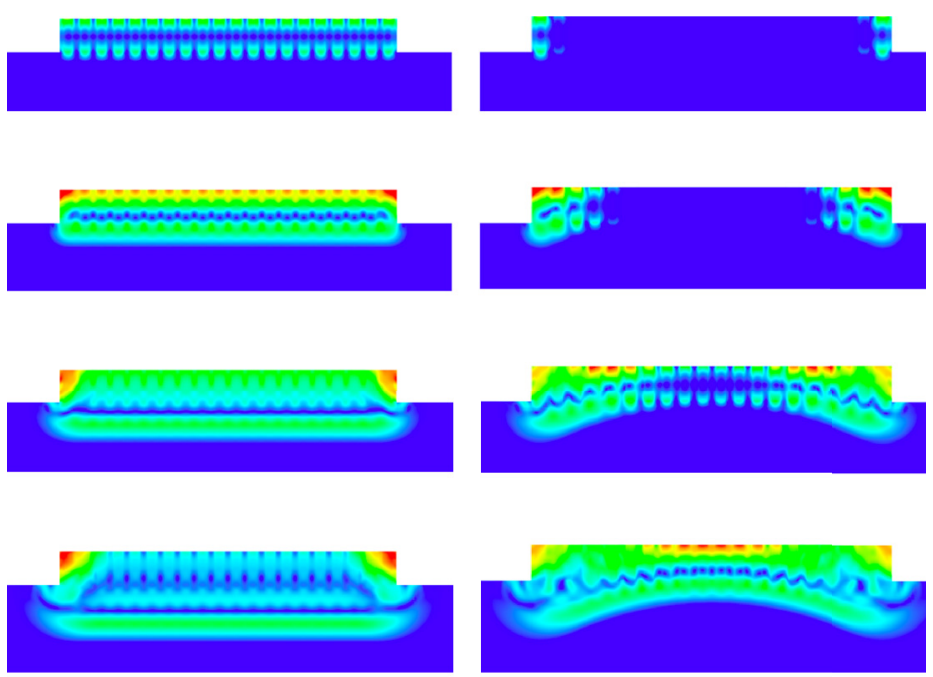

Figure 5. Top to bottom: snapshots of the absolute value of the displacement at $\mathrm{t}=0.26$, $0.52,0.78,1.04 \mu \mathrm{s}$. Zoom on the sensor when the piezoelectric bars are excited without delay (left) and with delay (right).
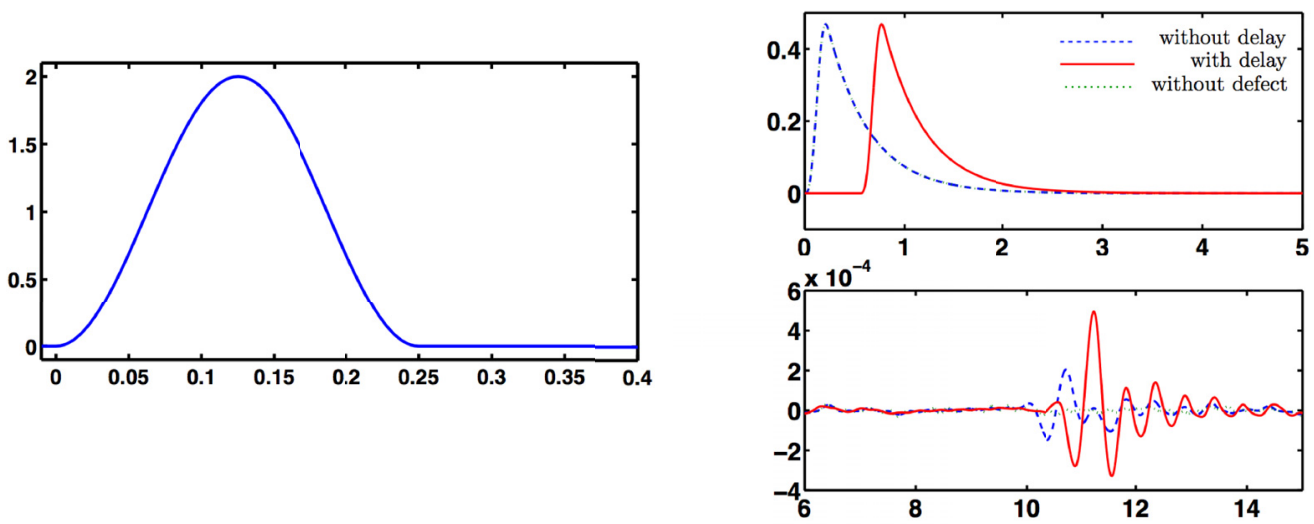

Figure 6. Value of the potential versus time for $V_{i}(t)$ with $\tau_{i}=0$ (left) and value of the potential versus time (in $\mu \mathrm{s}$ ) recovered on the tenth electrode (right). 

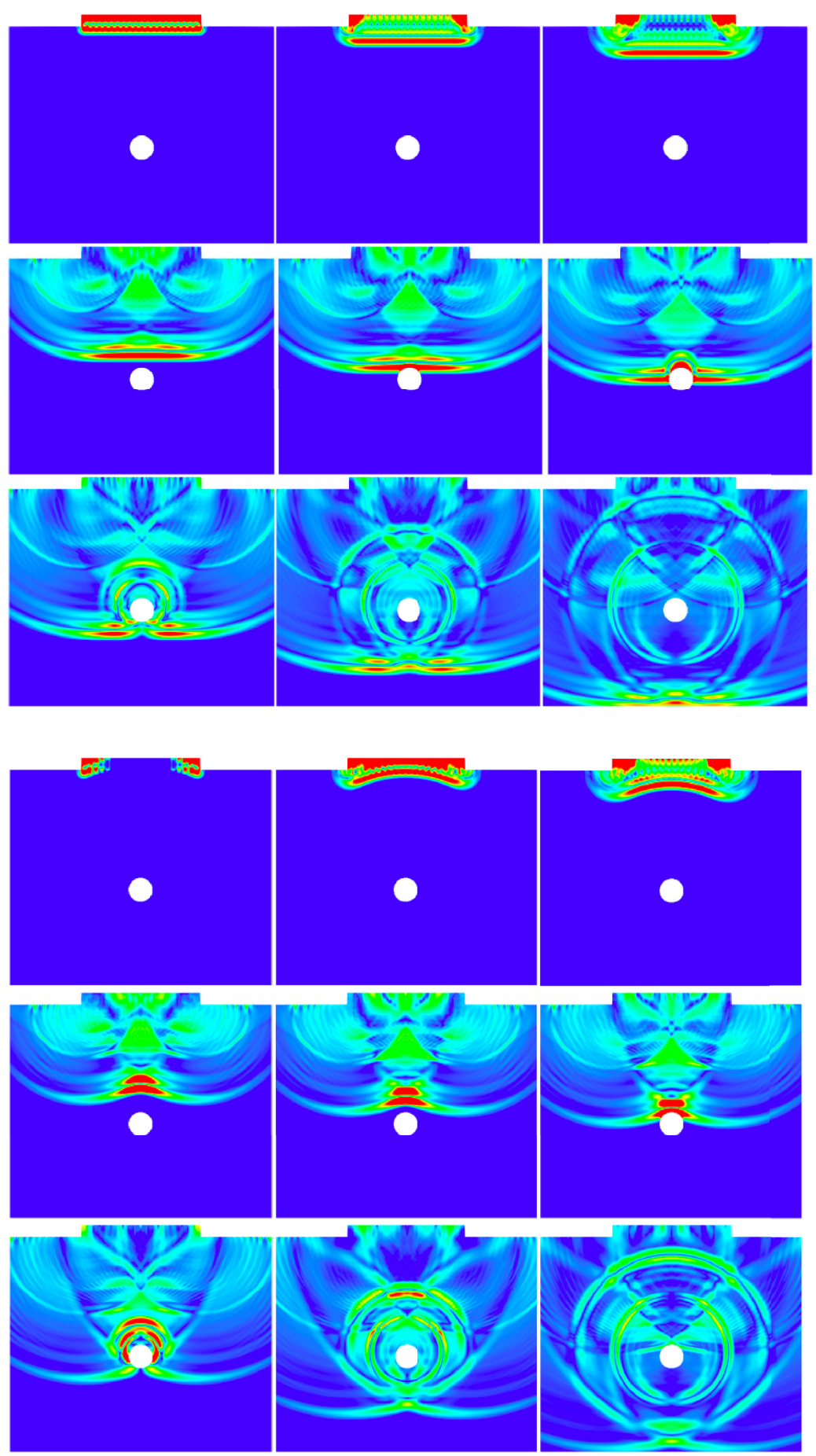

FigURE 7. From left to right, top to bottom: snapshots of the absolute value of the displacement at $t=0.52,1.04,1.57,4.71,5.24,5.76,6.81,8.39,9.96 \mu \mathrm{s}$. The electrodes are excited without delay (upper figures) and with delay (lower figures). 


\section{Appendix. Friedrichs Like inequality for FunCtions in $W_{\epsilon}\left(\mathbb{R}^{3}\right)$}

We recall the definition of the Hilbert space

$$
W_{\epsilon}\left(\mathbb{R}^{3}\right)=\left\{\frac{u}{\sqrt{1+|x|^{2}}} \in L^{2}\left(\mathbb{R}^{3}\right)^{3} \text { such that } \nabla \times u \in L^{2}\left(\mathbb{R}^{3}\right)^{3} \text { and } \nabla \cdot \epsilon u=0\right\},
$$

equipped with the norm

$$
\|u\|_{W\left(\mathbb{R}^{3}\right)}=\left\|\frac{u}{\sqrt{1+|\mathbf{x}|^{2}}}\right\|_{L^{2}\left(\mathbb{R}^{3}\right)}+\|\nabla \times u\|_{L^{2}\left(\mathbb{R}^{3}\right)}
$$

Theorem A.1. Let $u \in W_{\epsilon}\left(\mathbb{R}^{3}\right)$, there exists a constant $C>0$ such that:

$$
\left\|\frac{u}{\sqrt{1+|\mathbf{x}|^{2}}}\right\|_{L^{2}\left(\mathbb{R}^{3}\right)} \leq C\|\nabla \times u\|_{L^{2}\left(\mathbb{R}^{3}\right)},
$$

where $\epsilon$ is a positive scalar bounded piecewise smooth function of $x$ (the extension of this theorem when $\epsilon(x)$ is a matrix is trivial), constant outside the ball centered in 0 of radius $R>0: B_{0, R}$.

Proof. The idea is quite natural and consists in combining adequately local compactness argumentts for dealing with the region where the coefficient $\epsilon$ varies with the fact that "the div-curl norm" locally coincides with the $H^{1}$-norm, which we shall use in the exterior domain where the coefficient $\epsilon$ is constant.

In what follows $C$ will be the generic notation for a positive constant that may vary from one line to the other. The proof will be done by contradiction, assuming that the result if false, there exist a sequence of function $\left\{u_{n}\right\} \subset W_{\epsilon}\left(\mathbb{R}^{3}\right)$ such that

$$
\left\|\frac{u_{n}}{\sqrt{1+|x|^{2}}}\right\|_{L^{2}\left(\mathbb{R}^{3}\right)}=1, \quad\left\|\nabla \times u_{n}\right\|_{L^{2}\left(\mathbb{R}^{3}\right)}=\frac{1}{n} .
$$

$u_{n}$ being bounded in the norm of $W_{\epsilon}\left(\mathbb{R}^{3}\right)$ we have that $u_{n}$ converges weakly (up to a subsequence) to a limit $u$ which fulfills

$$
\|\nabla \times u\|_{L^{2}\left(\mathbb{R}^{3}\right)}=0
$$

since $u \in\left[L_{\text {loc }}^{2}\left(\mathbb{R}^{3}\right)^{3}\right.$ we have that $u=\nabla \varphi$ where $\varphi \in H_{l o c}^{1}\left(\mathbb{R}^{3}\right)$. As we also have $\nabla \cdot \epsilon u=0$ we have that $\varphi$ is solution of the problem

$$
-\nabla \cdot \epsilon \nabla \varphi=0, \quad x \in \mathbb{R}^{3}, \quad \varphi \in H_{l o c}^{1}\left(\mathbb{R}^{3}\right) \text { and } \frac{\nabla \varphi}{\sqrt{1+|x|^{2}}} \in L^{2}\left(\mathbb{R}^{3}\right)^{3},
$$

or equivalently ( $\mathbf{r}$ represent the unit vector normal to $B_{0,2 R}$ )

$$
-\nabla \cdot \epsilon \nabla \varphi=0, \quad x \in B_{0,2 R}, \quad \epsilon \nabla \varphi \cdot \mathbf{r}=T(\varphi), \quad x \in \partial B_{0,2 R},
$$

with the Dirichlet to Neumann operator $T$ defined by (see [16] for more details)

$$
\begin{array}{ccc}
T: H^{1 / 2}\left(\partial B_{0,2 R}\right) & \longmapsto & H^{-1 / 2}\left(\partial B_{0,2 R}\right) \\
\varphi & \longmapsto & T(\varphi)=(\epsilon \nabla \psi \cdot \mathbf{r})_{\mid \partial B_{0,2 R}},
\end{array}
$$

where $\psi$ is the solution of

$$
-\Delta \psi=0 \quad x \in \mathbb{R}^{3} \backslash B_{0,2 R}, \quad \psi=\varphi, \quad x \in \partial B_{0,2 R} .
$$


$\psi$ has an explicit expression (again see [16]) up to a constant term

$$
\psi=\sum_{l=0}^{+\infty} \sum_{m=-l}^{l} \frac{\left(\varphi, Y_{l}^{m}\right)_{L^{2}\left(\partial B_{0,2 R}\right)} Y_{l}^{m}}{\left((2 R)^{-1}|x|\right)^{l+1}},
$$

where $Y_{l}^{m}$ denote the spherical harmonic functions on the unit sphere. We can derive from equation (A.3) the expression of $T$ :

$$
T(\varphi)=-\sum_{l=0}^{+\infty} \sum_{m=-l}^{l}(l+1)\left(\varphi, Y_{l}^{m}\right)_{L^{2}\left(\partial B_{0,1}\right)} Y_{l}^{m} .
$$

Multiplying equation (A.2) by $\varphi$ gives the relation

$$
(\epsilon \nabla \varphi, \nabla \varphi)_{L^{2}\left(B_{0,2 R}\right)}-\langle T(\varphi), \varphi\rangle_{\partial B_{0,2 R}}=0,
$$

we can easily shown that $-\langle T(\varphi), \varphi\rangle_{\partial B_{0,1}}$ is positive for all $\varphi$, proving that $u=\nabla \varphi=0$ on $B_{0,2 R}$. We now introduce a smooth truncating function $\chi(x)$ which equals 1 on $B_{0, R}$ and 0 on $\mathbb{R}^{3} \backslash B_{0,2 R}$. We will denote by $\mathcal{O}$ the region $B_{0,2 R} \backslash B_{0, R}$. We have the decomposition

$$
u_{n}=v_{n}+w_{n} \text { with } v_{n}=\chi u_{n} \text { and } w_{n}=(1-\chi) u_{n},
$$

we naturally use the triangular inequality to write

$$
\left\|\frac{u_{n}}{\sqrt{1+|x|^{2}}}\right\|_{L^{2}\left(\mathbb{R}^{3}\right)} \leq\left\|\frac{v_{n}}{\sqrt{1+|\mathbf{x}|^{2}}}\right\|_{L^{2}\left(\mathbb{R}^{3}\right)}+\left\|\frac{w_{n}}{\sqrt{1+|x|^{2}}}\right\|_{L^{2}\left(\mathbb{R}^{3}\right)} .
$$

We have to bound $v_{n}$ and $w_{n}$ by terms in $u_{n}$ that must tends to 0 . For $v_{n}$ we have

$$
\left\|\frac{v_{n}}{\sqrt{1+|\mathbf{x}|^{2}}}\right\|_{L^{2}\left(\mathbb{R}^{3}\right)} \leq\left\|v_{n}\right\|_{L^{2}\left(B_{0,2 R}\right)} \leq C\left(\left\|\nabla \times v_{n}\right\|_{L^{2}\left(B_{0,2 R}\right)}+\left\|\nabla \cdot \epsilon v_{n}\right\|_{L^{2}\left(B_{0,2 R}\right)}\right),
$$

the second inequality is obtained using Friedrichs like inequality on domain satisfying the restricted cone property (smooth convex domain like $B_{0, R}$ satisfies the restricted cone property). This inequality is given by the following lemma:

Lemma A.1. Let $r_{n} \in L^{2}(\Omega)^{3}$ be a bounded sequence with $\nabla \cdot \epsilon r_{n} \in L^{2}(\Omega), \nabla \times r_{n} \in L^{2}(\Omega)$, where $\Omega$ respect the restricted cone property with $r \times n=0$ on $\partial \Omega$. Then there exist a limit $r$ and a subsequence $r_{m}$ such that $r_{m}$ converges strongly to $r$ in $L^{2}(\Omega)^{3}$. Moreover there exists $C>0$ such that for all $r \in L^{2}(\Omega)^{3}$ with $(\nabla \cdot \epsilon r, \nabla \times r) \in L^{2}(\Omega)$ and $r \times n=0$ on $\partial \Omega$ we have

$$
\|r\|_{L^{2}(\Omega)} \leq C\left(\|\nabla \cdot \epsilon r\|_{L^{2}(\Omega)}+\|\nabla \times r\|_{L^{2}(\Omega)}\right) .
$$

The proof is given in [19], the inequality being a direct consequence of the compactness result. This lemma imply that $v_{n}$ converges strongly to a limit $v$ in $L^{2}(\mathcal{O})^{3}$. The definition $v_{n}=\chi u_{n}$ implies that $u_{n}$ converges strongly to $u$ in $L^{2}(\mathcal{O})^{3}$ and so $u_{n}$ converges strongly to 0 in $L^{2}(\mathcal{O})^{3}$. Replacing $v_{n}$ in term of $u_{n}$ in the inequality (A.4) give

$$
\left\|\frac{v_{n}}{\sqrt{1+|\mathbf{x}|^{2}}}\right\|_{L^{2}\left(\mathbb{R}^{3}\right)} \leq C\left(\left\|\nabla \times u_{n}\right\|_{L^{2}\left(B_{0,2 R}\right)}+\|\nabla \chi\|_{L^{\infty}(\mathcal{O})}\left\|u_{n}\right\|_{L^{2}(\mathcal{O})}\right) .
$$

We now apply similar treatments on $w_{n}$. We first apply Hardy's inequality (again see [16]) to obtain

$$
\left\|\frac{w_{n}}{\sqrt{1+|\mathbf{x}|^{2}}}\right\|_{L^{2}\left(\mathbb{R}^{3}\right)} \leq\left\|\nabla w_{n}\right\|_{L^{2}\left(\mathbb{R}^{3}\right)}=\left\|\nabla w_{n}\right\|_{L^{2}\left(\mathbb{R}^{3 \times 3} \backslash B_{0, R}\right)} .
$$


We can decompose $\nabla w_{n}$ in a rotational part and a divergence part

$$
\begin{aligned}
\left\|\nabla w_{n}\right\|_{L^{2}\left(\mathbb{R}^{3 \times 3} \backslash B_{0, R}\right)} & =\left\|\nabla \times w_{n}\right\|_{L^{2}\left(\mathbb{R}^{3} \backslash B_{0, R}\right)}+\left\|\nabla \cdot w_{n}\right\|_{L^{2}\left(\mathbb{R} \backslash B_{0, R}\right)} \\
& \leq C\left(\left\|\nabla \times w_{n}\right\|_{L^{2}\left(\mathbb{R}^{3} \backslash B_{0, R}\right)}+\left\|\nabla \cdot \epsilon w_{n}\right\|_{L^{2}\left(\mathbb{R}^{3} \backslash B_{0, R}\right)}\right) .
\end{aligned}
$$

The inequality comes from the fact that we introduced the constant $\epsilon$ in the norm of the divergence of $w_{n}$. Finally by writing $w_{n}$ in term of $u_{n}$ we have

$$
\left\|\frac{w_{n}}{\sqrt{1+|\mathbf{x}|^{2}}}\right\|_{L^{2}\left(\mathbb{R}^{3}\right)^{3}} \leq C\left(\left\|\nabla \times u_{n}\right\|_{L^{2}\left(\mathbb{R}^{3} \backslash B_{0, R}\right)^{3}}+\|\nabla \chi\|_{L^{\infty}(\mathcal{O})^{3}}\left\|u_{n}\right\|_{L^{2}(\mathcal{O})^{3}}\right),
$$

we can add (A.5) and (A.8) to obtain

$$
\left\|\frac{u_{n}}{\sqrt{1+|\mathbf{x}|^{2}}}\right\|_{L^{2}\left(\mathbb{R}^{3}\right)^{3}} \leq C\left(\left\|\nabla \times u_{n}\right\|_{L^{2}\left(\mathbb{R}^{3}\right)^{3}}+\|\nabla \chi\|_{L^{\infty}(\mathcal{O})^{3}}\left\|u_{n}\right\|_{L^{2}(\mathcal{O})^{3}}\right),
$$

as $\left\|u_{n}\right\|_{L^{2}(\mathcal{O})}$ tends strongly to 0 with $n$, we obtain a contradiction.

\section{REFERENCES}

[1] N. Abboud, G. Wojcik and D.K. Vaughan, Finite element modeling for ultrasonic transducers. SPIE Int. Symp. Medical Imaging (1998).

[2] E. Canon and M. Lenczner, Models of elastic plates with piezoelectric inclusions part i: Models without homogenization. Math. Comput. Model. 26 (1997) 79-106.

[3] P. Challande, Optimizing ultrasonic transducers based on piezoelectric composites using a finite-element method. IEEE Trans. Ultrason. Ferroelectr. Freq. Control 37 (2002) 135-140.

[4] G.C. Cohen, Higher-order numerical methods for transient wave equations. Springer (2002).

[5] E. Dieulesaint and D. Royer, Elastic waves in solids, free and guided propagation. Springer (2000).

[6] M. Durufle, P. Grob and P. Joly, Influence of gauss and gauss-lobatto quadrature rules on the accuracy of a quadrilateral finite element method in the time domain. Numer. Methods Partial Differ. Equ. 25 (2009) 526-551.

[7] Y. Gómez-Ullate Ricón and F.M. de Espinosa Freijo, Piezoelectric modelling using a time domain finite element program. J. Eur. Ceram. Soc. 27 (2007) 4153-4157.

[8] T. Ikeda, Fundamentals of piezoelectricity. Oxford science publications (1990).

[9] N.A. Kampanis, V.A. Dougalis and J.A. Ekaterinaris, Effective computational methods for wave propagation. Chapman and Hall/CRC (2008).

[10] T. Lahrner, M. Kaltenbacher, B. Kaltenbacher, R. Lerch and E. Leder. Fem-based determination of real and complex elastic, dielectric, and piezoelectric moduli in piezoceramic materials. IEEE Trans. Ultrason. Ferroelectr. Freq. Control 55 (2008) $465-475$.

[11] R. Lerch, Simulation of piezoelectric devices by two-and three-dimensional finite elements. IEEE Trans. Ultrason. Ferroelectr. Freq. Control 37 (2002) 233-247.

[12] S. Li, Transient wave propagation in a transversely isotropic piezoelectric half space. Z. Angew. Math. Phys. 51 (2000) $236-266$.

[13] D. Mercier and S. Nicaise, Existence, uniqueness, and regularity results for piezoelectric systems. SIAM J. Math. Anal. 37 (2005) 651-672.

[14] J. San Miguel, J. Adamowski and F. Buiochi, Numerical modeling of a circular piezoelectric ultrasonic transducer radiating in water. ABCM Symposium Series in Mechatronics 2 (2005) 458-464.

[15] P. Monk, Finite element methods for maxwell's equations. Oxford science publications (2003).

[16] J.C. Nédélec, Acoustic and electromagnetic equations: integral representations for harmonic problems. Springer (2001).

[17] V. Priimenko and M. Vishnevskii, An initial boundary-value problem for model electromagnetoelasticity system. J. Differ. Equ. 235 (2007) 31-55.

[18] L. Schmerr Jr and S.J. Song, Ultrasonic nondestructive evaluation systems. Springer (2007).

[19] C. Weber and P. Werner, A local compactness theorem for maxwell's equations. Math. Methods Appl. Sci. 2 (1980) 12-25. 